\title{
XMM-NEWTON X-RAY AND ULTRAVIOLET OBSERVATIONS OF THE FAST NOVA V2491 Cyg DURING THE SUPERSOFT SOURCE PHASE
}

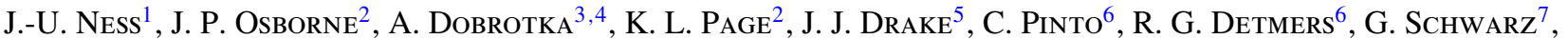 \\ M. F. Bode ${ }^{8}$, A. P. Beardmore ${ }^{2}$, S. Starrfield ${ }^{9}$, M. HernanZ ${ }^{10}$, G. Sala ${ }^{11}$, J. Krautter ${ }^{12}$, and C. E. Woodward ${ }^{13}$ \\ ${ }^{1}$ XMM-Newton Science Operations Centre, ESA, Madrid, Spain \\ ${ }^{2}$ Department of Physics \& Astronomy, University of Leicester, Leicester, LE1 7RH, UK \\ ${ }^{3}$ Department of Physics, Institute of Materials Science, Faculty of Materials Science and Technology, Slovak University of Technology in Bratislava, \\ 91724 Trnava, Slovakia \\ ${ }^{4}$ Department of Astronomy, Graduate School of Science, Kyoto University, Sakyo-ku, Kyoto 606-8502, Japan \\ ${ }^{5}$ Harvard-Smithsonian Center for Astrophysics, Cambridge, MA 02138, USA \\ ${ }^{6}$ SRON Netherlands Institute for Space Research, 3584 CA Utrecht, The Netherlands \\ ${ }^{7}$ American Astronomical Society, Washington, DC 20009-1231, USA \\ ${ }^{8}$ Astrophysics Research Institute, Liverpool John Moores University, Birkenhead CH41 1LD, UK \\ ${ }^{9}$ School of Earth and Space Exploration, Arizona State University, Tempe, AZ 85287-1404, USA \\ ${ }^{10}$ Institut de Ciències de l'Espai (CSIC-IEEC), Campus UAB, Facultat de Ciències, 08193 Bellaterra (Barcelona), Spain \\ ${ }^{11}$ Departament Física i Enginyeria Nuclear, EUETIB (UPC-IEEC), 08036 Barcelona, Spain \\ ${ }^{12}$ Landessternwarte Königstuhl, Universität Heidelberg, D-69117 Heidelberg, Germany \\ ${ }^{13}$ Department of Astronomy, University of Minnesota, Minneapolis, MN 55455, USA \\ Received 2010 November 7; accepted 2011 March 21; published 2011 May 5
}

\begin{abstract}
Two XMM-Newton observations of the fast classical nova V2491 Cyg were carried out in short succession on days 39.93 and 49.62 after discovery, during the supersoft source (SSS) phase, yielding simultaneous X-ray and UV light curves and high-resolution X-ray spectra. The first X-ray light curve is highly variable, showing oscillations with a period of 37.2 minutes after an extended factor of three decline lasting $\sim 3 \mathrm{hr}$, while the second X-ray light curve is less variable. The cause of the dip is currently unexplained and has most likely the same origin as similar events in the early SSS light curves of the novae V4743 Sgr and RS Oph, as it occurred on the same timescale. The oscillations are not present during the dip minimum and also not in the second observation. The UV light curves are variable but contain no dips and no period. High-resolution X-ray spectra are presented for four intervals of differing intensity. All spectra are atmospheric continua with deep absorption lines and absorption edges. Two interstellar lines of $\mathrm{O}_{\mathrm{I}}$ and $\mathrm{N}_{\mathrm{I}}$ are clearly seen at their rest wavelengths, while a large number of high-ionization absorption lines are found at blueshifts indicating an expansion velocity of $3000-3400 \mathrm{~km} \mathrm{~s}^{-1}$, which does not change significantly during the epochs of observation. Comparisons with the slower nova V4743 Sgr and the symbiotic recurrent nova RS Oph are presented. The SSS spectrum of V4743 Sgr is much softer with broader and more complex photospheric absorption lines. The ejecta are extended, allowing us to view a larger range of the radial velocity profile. Meanwhile, the absorption lines in RS Oph are as narrow as in V2491 Cyg, but they are less blueshifted. A remarkable similarity in the continua of V2491 Cyg and RS Oph is found. The only differences are smaller line shifts and additional emission lines in RS Oph that are related to the presence of a dense stellar wind from the evolved companion. Three unidentified absorption lines are present in the X-ray spectra of all three novae, with projected rest wavelengths $26.05 \AA$, $29.45 \AA$, and $30.0 \AA$. No entirely satisfactory spectral model is currently available for the soft X-ray spectra of novae in outburst, and careful discussion of assumptions is required.
\end{abstract}

Key words: astronomical databases: miscellaneous - novae, cataclysmic variables - stars: individual (V2491 Cyg, RS Oph, V4743 Sgr)

Online-only material: color figures

\section{INTRODUCTION}

Classical nova $(\mathrm{CN})$ and recurrent nova $(\mathrm{RN})$ outbursts result from nuclear explosions on the surface of a white dwarf (WD) that has accreted hydrogen-rich material from a companion star, which in the case of most $\mathrm{CNe}$ is a low-mass mainsequence star. Some RNe occur in symbiotic systems where the companion is evolved. Enough energy is produced to eject the outer envelope. The radiative output of the nova first appears primarily in the optical, but as the density in the ejecta drops as a consequence of decreasing mass-loss rate from the WD surface and continued expansion, the radius of the pseudo-photosphere shrinks, and successively hotter layers become visible (see, e.g., Gallagher \& Starrfield 1978; Hauschildt et al. 1992). If nuclear burning continues long enough, the peak of the spectral energy distribution (SED) eventually shifts into the $\mathrm{X}$-ray regime, and at that time the nova emits an X-ray spectrum that resembles those typically observed in the class of supersoft X-ray sources (SSS; Kahabka \& van den Heuvel 1997). For a review of nova evolution see, e.g., Bode \& Evans (2008).

In the standard picture of nova evolution, the only time at which novae are bright X-ray emitters is during this SSS phase, but it is difficult to predict at which time this phase starts. In an attempt to catch a nova during the SSS phase, early observations have to be taken that bear the risk of no detection. During these campaigns, several novae have been observed before the SSS phase started, yielding faint, hard X-ray spectra (e.g., Lloyd et al. 1992; Krautter et al. 1996; Mukai \& Ishida 2001; Orio et al. 2001). The early faint, hard X-ray emission is believed to arise from shocks in the ejecta or in specific cases such as 
RS Oph and other symbiotic systems, in shocks between the ejecta and the dense stellar wind of the companion. In very special cases such as V458 Vul, early hard emission may also arise from interactions of the ejecta with surrounding material in a pre-existing planetary nebula (Wesson et al. 2008; Ness et al. 2009a, and references therein).

The uncertainty of the times at which bright SSS emission can be seen has been significantly reduced by systematic monitoring with the X-ray Telescope (XRT) on board the Swift observatory. The first dense Swift monitoring campaign was carried out after the 2006 outburst of RS Oph (Bode et al. 2006; Osborne et al. 2011; Page et al. 2008), and XMM-Newton and Chandra observations were scheduled at times that were strategically important (e.g., Ness et al. 2007, 2009b).

V2491 Cyg was discovered on 2008 April 10.728 UT by Nakano et al. (2008). The optical brightness was 7.7 mag at the time of discovery and it reached a peak magnitude of $7.5 \mathrm{mag}$ one day later. The timescale by which the $V$ brightness decreased by 2 mag was $t_{2}=4.6$ days (Tomov et al. 2008a), as such a very fast nova. A rebrightening was observed after JD $\sim 2454575$ (April 26). Naik et al. (2009) discussed this secondary peak. In early spectroscopic observations, P-Cygni line profiles were found with a velocity of about $-4000 \mathrm{~km} \mathrm{~s}^{-1}$ in $\mathrm{H} \alpha$ and $\mathrm{H} \beta$ profiles (Tomov et al. 2008b). Near-infrared (NIR) photometry (Naik et al. 2009) and spectroscopy (Rudy et al. 2008) obtained in 2008 April showed no sign of dust formation. Unpublished NIR spectroscopy taken in 2008 July also revealed no dust formation (R. J. Rudy 2010, private communication), indicating that V2491 Cyg was not a dust-forming nova. Rudy et al. (2008) measured $E(B-V)=0.43$, which converts to an interstellar neutral hydrogen column density of $N_{\mathrm{H}}=2.6 \times 10^{21} \mathrm{~cm}^{-2}$ using the relation $\left\langle N_{\mathrm{H}} / E(B-V)\right\rangle=(6 \pm 2) \times 10^{21} \mathrm{~cm}^{-2}$ (Dickey \& Lockman 1990; Bohlin et al. 1978).

The distance was estimated to be $10.5 \mathrm{kpc}$ by Helton et al. (2008) and, more recently, $14 \mathrm{kpc}$ by Munari et al. (2011), both based on a Maximum Magnitude versus Rate of Decline (MMRD) relation by della Valle \& Livio (1995) and Downes \& Duerbeck (2000). We note, however, that in addition to the high uncertainty of this method (see, e.g., Warner 2008), a second, smaller, maximum occurred about three weeks after discovery, which reduces the confidence of this method. This makes the time zero from which the MMRD relation should be used uncertain.

In X-rays, V2491 Cyg was observed with Swift 1.02 days after discovery, but only a marginal detection was achieved (Page et al. 2010), which could, however, be contaminated by optical loading, i.e., the high number of optical photons leads to false registration of X-ray events (Ibarra \& Kuulkers 2008). During a second Swift observation on day 4.56 after discovery, a count rate of $0.009 \pm 0.002$ counts per second (cps) was reported by Kuulkers et al. (2008). In a Suzaku observation taken nine days after discovery, superhard X-ray emission extending out to $70 \mathrm{keV}$ was detected in addition to a hard thermal spectrum (Takei et al. 2009).

This nova is only the second nova to be detected in X-rays before the outburst, after V2487 Oph (Hernanz \& Sala 2002). Ibarra et al. (2009) reported on five Swift pre-outburst observations in which V2491 Cyg was serendipitously in the field of view of the XRT with clear detection.

Based on the optical spectral characteristics of V2491 Cyg, Tomov et al. (2008a) speculated that it is an RN, similar to U Sco and V394 CrA. The similarity to V2487 Oph with regard to preoutburst X-ray emission and the identification of V2487 Oph as

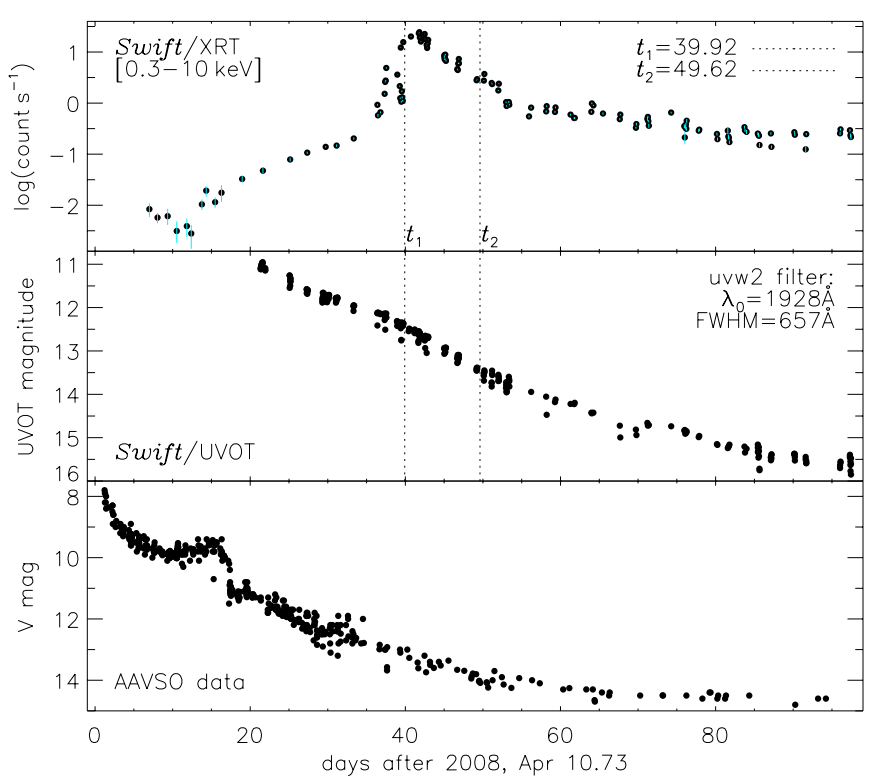

Figure 1. Swift X-ray (top) and UV (middle) light curves from Page et al. (2010) with times of XMM-Newton observation marked by vertical dotted lines. In the bottom panel, the AAVSO light curve is added, showing the evolution in the $V$ filter. A small drop in X-ray count rate a few days before the secondary peak can be seen.

an RN by Pagnotta et al. (2009; they discovered a prior outburst in 1900) support this speculation.

The evolution of this nova was followed with Swift observations every one to three days. Page et al. (2010) give a detailed description of the evolution of X-ray brightness, X-ray spectra, and UV brightness.

The Swift monitoring observations were used to schedule two XMM-Newton observations as illustrated in Figure 1 (Ness et al. 2008a, 2008b), where, from top to bottom, light curves are shown from Swift/XRT and UVOT data taken from Page et al. (2010), and $V$ filter magnitudes from the database of the American Association of Variable Star Observers (AAVSO). The secondary peak can be seen in the AAVSO light curve around day 15, a few days after a small reduction in the X-ray brightness.

The first XMM-Newton observation was carried out five days after the first detection of SSS emission. As soon as the indication of a decline was found, a second XMM-Newton observation was obtained. A rich data set was acquired, including X-ray and UV light curves, high-resolution X-ray spectra in the energy range $0.3-2 \mathrm{keV}$, and low-resolution $\mathrm{X}$-ray spectra in the range $0.1-10 \mathrm{keV}$. In this paper, the soft X-ray spectra and X-ray and UV light curves are presented and described. In Section 2, we describe the observations and the data reduction. The light curves are presented and analyzed in Section 3, and the presentation of X-ray spectra can be found in Section 4. These descriptions need to be complemented by models that will be developed and discussed by theoretical working groups and are therefore not discussed beyond best fits of publicly available models in Section 4.4. The results from light curve and spectral analyses are discussed in Section 5 with a focus on spectral modeling, comparison with X-ray spectra of other novae, and highamplitude variations. Summary and conclusions are given in Section 6.

\section{OBSERVATIONS}

Two XMM-Newton observations of V2491 Cyg were taken, starting on 2008 May 20.6 (39.93 days after discovery) and May 
Table 1

Observation Log

\begin{tabular}{|c|c|c|c|c|c|c|}
\hline \multirow{2}{*}{$\begin{array}{l}\text { Instrument } \\
\text { ObsID } 0552270501\end{array}$} & \multirow[t]{2}{*}{ Mode } & \multirow[t]{2}{*}{ Filter } & Start Time & End Time & \multirow{2}{*}{$\begin{array}{l}\text { Scheduled } \\
\text { Duration } \\
\text { (s) }\end{array}$} & \multirow{2}{*}{$\begin{array}{l}\text { Performed } \\
\text { Duration } \\
\text { (s) }\end{array}$} \\
\hline & & & \multicolumn{2}{|c|}{ YYYY-MM-DD@HH:MM:SS } & & \\
\hline MOS1 & Small Window & MEDIUM & 2008-05-20@14:04:42 & 2008-05-21@00:58:39 & 39057 & 39057 \\
\hline MOS2 & Small Window & MEDIUM & 2008-05-20@14:04:42 & 2008-05-21@00:58:44 & 39062 & 39062 \\
\hline pn & Timing & MEDIUM & 2008-05-20@14:24:03 & 2008-05-21@00:58:59 & 38036 & 38036 \\
\hline RGS1 & Spectroscopy SES & & 2008-05-20@14:03:29 & 2008-05-21@00:59:54 & 39283 & 39283 \\
\hline RGS2 & Spectroscopy SES & & 2008-05-20@14:03:34 & 2008-05-21@00:59:54 & 39278 & 39278 \\
\hline $\mathrm{OM}$ & & UVW1 & 2008-05-20@14:09:10 & 2008-05-20@15:19:16 & 3900 & 3900 \\
\hline $\mathrm{OM}$ & & UVW1 & 2008-05-20@15:19:17 & 2008-05-20@17:07:43 & 4400 & 4400 \\
\hline $\mathrm{OM}$ & & UVW1 & 2008-05-20@17:07:44 & 2008-05-20@18:26:10 & 4400 & $0^{\mathrm{a}}$ \\
\hline $\mathrm{OM}$ & & UVM2 & 2008-05-20@18:26:11 & 2008-05-20@19:44:37 & 4400 & $0^{\mathrm{a}}$ \\
\hline $\mathrm{OM}$ & & UVM2 & 2008-05-20@19:44:38 & 2008-05-20@21:03:04 & 4400 & 4400 \\
\hline $\mathrm{OM}$ & & UVM2 & 2008-05-20@21:03:05 & 2008-05-20@22:21:31 & 4400 & 4400 \\
\hline $\mathrm{OM}$ & & UVW2 & 2008-05-20@22:21:32 & 2008-05-20@23:39:58 & 4400 & 4400 \\
\hline OM & & UVW2 & 2008-05-20@23:39:59 & 2008-05-21@00:58:25 & 4400 & 4400 \\
\hline \multicolumn{7}{|l|}{ ObsID 0552270601} \\
\hline MOS1 & Small Window & THIN 1 & 2008-05-30@08:21:53 & 2008-05-30@16:39:10 & 29657 & 31150 \\
\hline MOS2 & Small Window & THIN 1 & 2008-05-30@08:21:53 & 2008-05-30@16:39:15 & 29662 & 31165 \\
\hline pn & Timing & MEDIUM & 2008-05-30@08:41:14 & 2008-05-30@16:39:30 & 28636 & 30208 \\
\hline RGS1 & Spectroscopy SES & & 2008-05-30@08:20:40 & 2008-05-30@16:40:25 & 29883 & 31725 \\
\hline RGS2 & Spectroscopy SES & & 2008-05-30@08:20:45 & 2008-05-30@16:40:25 & 29878 & 31657 \\
\hline $\mathrm{OM}$ & & UVW1 & 2008-05-30@08:26:21 & 2008-05-30@09:41:27 & 4200 & 4200 \\
\hline $\mathrm{OM}$ & & UVW1 & 2008-05-30@09:41:28 & 2008-05-30@10:59:54 & 4400 & 4400 \\
\hline $\mathrm{OM}$ & & UVM2 & 2008-05-30@10:59:55 & 2008-05-30@12:13:21 & 4100 & 4100 \\
\hline $\mathrm{OM}$ & & UVM2 & 2008-05-30@12:13:22 & 2008-05-30@13:31:48 & 4400 & 4400 \\
\hline $\mathrm{OM}$ & & UVW2 & 2008-05-30@13:31:49 & 2008-05-30@14:50:15 & 4400 & 4400 \\
\hline $\mathrm{OM}$ & & UVW2 & 2008-05-30@14:50:16 & 2008-05-30@16:38:42 & 4400 & $0^{\mathrm{a}}$ \\
\hline
\end{tabular}

Note. ${ }^{\text {a }}$ Double bit memory errors.

30.3 (49.62 days after discovery). The XMM-Newton observatory consists of five different instruments behind three mirrors plus an optical monitor (OM) that all observe simultaneously. The observation details are listed in Table 1.

For this paper, only the spectra from the Reflection Grating Spectrometers (RGS; den Herder et al. 2001), the light curves from the EPIC/pn, and the OM (Talavera 2009) are used. As will be shown, the SSS spectra range from 15 to $38 \AA$ $(0.33-0.83 \mathrm{keV})$, a range completely covered by the RGS. Since the RGS spectrum is sufficiently well exposed for our analysis, the EPIC spectra of this spectral range are not needed. At energies above $2 \mathrm{keV}$, optically thin thermal emission is observable in the EPIC spectra. These are discussed in the context of additional Suzaku observations by Takei et al. (2011).

The RGS consists of two identical grating spectrometers, RGS1 and RGS2, behind different mirrors. The dispersed photons are recorded by a strip of eight CCD metal oxide semiconductor (MOS) chips. One of these chips has failed in each spectrometer leading to gaps in the spectra which fortunately affect different spectral regions, and the missing information can be retrieved from the other spectrometer. A number of spectral bins contain no information because of bad pixels. Again, the redundancy of having two spectrometers allows almost all gaps to be filled.

The OM was operated in Science User Defined imaging plus fast mode, which yields UV light curves. Several shorter exposures were taken with the UVW1 $(\lambda \sim 2500-3500 \AA)$, UVM2 $(\lambda \sim 2000-2600 \AA)$, and UVW2 $(\lambda \sim 1900-2300 \AA)$ filters. Some of the OM exposures failed because of double bit memory errors (listed with 0 exposure time in Table 1). These exposures are not recoverable.
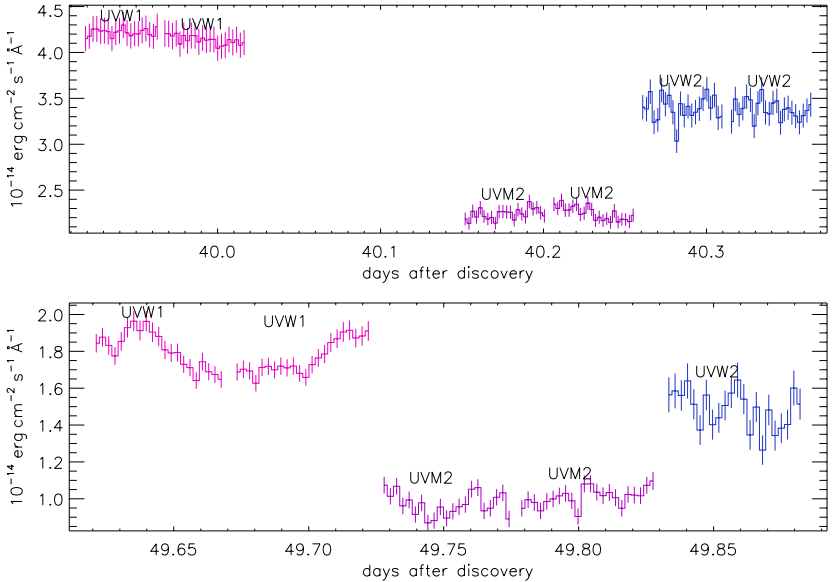

Figure 2. OM light curves in flux units. The time axis is plotted in units of fractional day after discovery (2008 April 10.728). The time range between days 40.02 and 41.15 are not covered because of double bit memory problems leading to unrecoverable loss of data.

(A color version of this figure is available in the online journal.)

In Table 2, the average magnitudes and fluxes in each filter are listed for the exposures taken at the times given in the first column. The UVW2 magnitudes are consistent with the Swift/ UVOT measurements reported by Page et al. (2010) for the corresponding times. A graphical illustration of OM fluxes is shown in Figure 2.

The EPIC/pn was operated in timing mode. The events were all collapsed into a single column, allowing fast readout of the chips. While the high time resolution is not critical to our analysis, the faster readout prevents pile up, and the extracted 
Table 2

Ultraviolet Magnitudes and Fluxes for Three OM Filters

\begin{tabular}{|c|c|c|c|c|c|c|}
\hline \multirow[t]{2}{*}{ Day } & \multicolumn{2}{|c|}{$\begin{array}{c}\text { UVW1 } \\
\lambda_{\text {eff }}^{\mathrm{a}}=2910 \AA\end{array}$} & \multicolumn{2}{|c|}{$\begin{array}{c}\text { UVM2 } \\
\lambda_{\text {eff }}^{\mathrm{a}}=2310 \AA\end{array}$} & \multicolumn{2}{|c|}{$\begin{array}{c}\text { UVW2 } \\
\lambda_{\mathrm{eff}}^{\mathrm{a}}=2120 \AA\end{array}$} \\
\hline & (mag) & $(\text { flux })^{b}$ & (mag) & $(\text { flux })^{b}$ & (mag) & $(\text { flux })^{b}$ \\
\hline 39.93 & 12.29 & $4.39 \pm 0.005$ & & & & \\
\hline 39.98 & 12.30 & $4.36 \pm 0.013$ & & & & \\
\hline 40.16 & & & 13.17 & $2.42 \pm 0.01$ & & \\
\hline 40.21 & & & 13.17 & $2.42 \pm 0.01$ & & \\
\hline 40.27 & & & & & 12.89 & $3.53 \pm 0.021$ \\
\hline 40.32 & & & & & 12.90 & $3.50 \pm 0.021$ \\
\hline 49.63 & 13.21 & $1.89 \pm 0.004$ & & & & \\
\hline 49.68 & 13.23 & $1.85 \pm 0.004$ & & & & \\
\hline 49.73 & & & 14.06 & $1.06 \pm 0.013$ & & \\
\hline 49.78 & & & 14.03 & $1.10 \pm 0.012$ & & \\
\hline 49.84 & & & & & 13.79 & $1.54 \pm 0.024$ \\
\hline
\end{tabular}

Notes.

a Effective wavelength.

b $10^{-14} \mathrm{erg} \mathrm{cm}^{-2} \mathrm{~s}^{-1} \AA^{-1}$.

spectra and light curves can be used without non-standard corrections.

We have used standard Science Analysis Software (SAS, version 10.0) tools for the reduction of light curves and spectra. The new SAS tool xmmextractor was particularly helpful in determining optimized extraction regions and the correction of telemetry losses in the EPIC/pn light curve. ${ }^{14}$

\section{LIGHT CURVES}

In Figure 3, we present the X-ray light curves extracted from the RGS1 in comparison with the simultaneously taken ultraviolet light curves shown in Figure 2. The UV fluxes are shifted and rescaled to fit in the sample plot. The time axis is plotted in units of fractional day after discovery (2008 April 10.728).

In the first observation, starting 39.93 days after discovery, the X-ray light curve is highly variable, while the UV light curves appear less variable. We have checked for variability in the light curves in both bands. For each OM exposure, we tested the original (non-scaled) count rate, to be constant at the median value and calculated a value of reduced $\chi_{v}^{2}$. All exposures yield $\chi_{v}^{2} \sim 1$, indicating no significant variability longer than the bin size of $50 \mathrm{~s}$. The most prominent event in the X-ray light curve is an extended dip in count rate, commencing on day 40.01, lasting 0.12 days $(2.9 \mathrm{hr})$. After this event, the X-ray count rate rises again, yielding a higher count rate than before the dip. Unfortunately, two OM exposures failed during the most interesting time interval between 40.02 and 40.15 days. We are therefore unable to study any relations between the dip in X-rays and the UV. In addition to the dip, shorter variations of order 15 minutes can be seen in the X-ray count rate before day 40.0 and after day 40.12 which could be oscillations (see Section 3.1 below). We fitted a fifth-order polynomial to the entire RGS1 $\mathrm{X}$-ray light curve. Although the polynomial describes the longterm trend well, we only found a best-fit value of $\chi_{v}^{2}=10.3$ (with 781 degrees of freedom), thus additional variability on shorter timescales is present at a significant level. Interestingly, during the dip, no oscillations seem to be present which we verified by computing $\chi_{v}^{2}$ for the time interval during the dip, finding $\chi_{v}^{2}<1$ after fitting a fifth-order polynomial to this

\footnotetext{
14 Many thanks to Aitor Ibarra for support with xmmextractor.
}
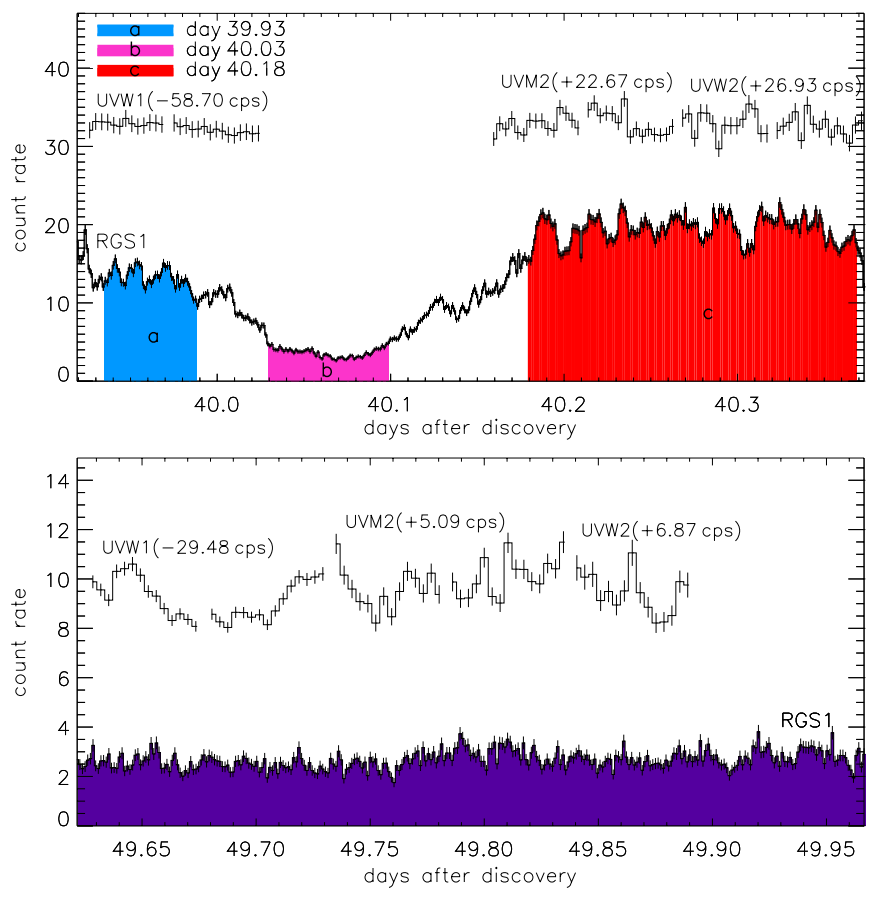

Figure 3. X-ray RGS1 and OM light curves for the observations starting on days 39.93 (top) and 49.62 (bottom). Time bin sizes are $50 \mathrm{~s}$ and $300 \mathrm{~s}$ for RGS1 and OM, respectively. The OM count rates were rescaled to fit in the graph. The shaded areas mark time intervals over which we have extracted separate RGS spectra for studies of spectral changes with the photometric variability (see Table 3).

(A color version of this figure is available in the online journal.)

subset as well as for a constant count rate. For the part after the dip, we find $\chi_{v}^{2}=7.2$ (340 degrees of freedom) when compared to a fifth-order polynomial, a bit better, but indicating that there is more variability in addition to the dip, motivating a detailed analysis of variability in Section 3.1.

The shaded regions indicate three time intervals for which we have extracted RGS spectra that are discussed in Section 4. We refer to these episodes as phases a, b, and c as indicated inside the shaded areas and the legend (see also Table 3 ). Phase $\mathrm{a}$ is the pre-dip spectrum, phase $\mathrm{b}$ corresponds to the time of low-flux emission, and phase $\mathrm{c}$ is the post-dip. 
Table 3

Separation of RGS Spectra into Four Time Segments with Blackbody and Atmosphere Model Parameters

\begin{tabular}{|c|c|c|c|c|c|c|c|c|c|c|c|c|c|}
\hline $\begin{array}{l}\text { Start } \\
\text { Date }^{\mathrm{a}} \\
\end{array}$ & $\begin{array}{l}\text { Color } \\
\text { Code }\end{array}$ & $\begin{array}{l}\Delta t^{\mathrm{b}} \\
(\mathrm{ks})\end{array}$ & flux $^{\mathrm{c}}$ & $\begin{array}{c}T_{\mathrm{BB}}{ }^{\mathrm{d}} \\
\left(10^{5} \mathrm{~K}\right)\end{array}$ & $N_{\mathrm{H}}^{\mathrm{d}, \mathrm{e}}$ & $\begin{array}{c}R_{\mathrm{BB}}{ }^{\mathrm{d}} \\
\left(10^{8} \mathrm{~cm}\right)\end{array}$ & $A_{\mathrm{O}}^{\mathrm{d}, \mathrm{f}}$ & $\begin{array}{c}T_{\mathrm{eff}^{\mathrm{g}}}^{\mathrm{g}} \\
\left(10^{5} \mathrm{~K}\right)\end{array}$ & $N_{\mathrm{H}}^{\mathrm{e}, \mathrm{g}}$ & $\begin{array}{c}R_{\mathrm{eff}^{\mathrm{g}}} \\
\left(10^{8} \mathrm{~cm}\right)\end{array}$ & $\begin{array}{c}\log \left(L_{\mathrm{bol}}\right)^{\mathrm{g}} \\
\left(\mathrm{erg} \mathrm{s}^{-1}\right)\end{array}$ & $\begin{array}{c}M_{\mathrm{WD}}{ }^{\mathrm{g}} \\
\left(M_{\odot}\right)\end{array}$ & $A_{\mathrm{O}}^{\mathrm{f}, \mathrm{g}}$ \\
\hline 39.93 & Blue (a) & 4.7 & 3.57 & 5.9 & 4.9 & 77 & 1.6 & $9.6-10.3$ & $2.1-2.4$ & $5.7-7.3$ & $38.42-38.52$ & $2.48-4.18$ & $3.4-4.1$ \\
\hline 40.03 & Pink (b) & 6.1 & 0.98 & 6.3 & 4.1 & 22 & 2.0 & $9.8-10.5$ & $1.6-1.8$ & $2.5-3.1$ & $37.73-37.81$ & $0.46-0.72$ & $4.7-5.4$ \\
\hline 40.18 & $\operatorname{Red}(\mathrm{c})$ & 16.5 & 5.09 & 6.3 & 4.7 & 57 & 1.2 & $9.7-10.4$ & $2.0-2.3$ & $5.8-7.8$ & $38.45-38.58$ & $2.54-4.34$ & $2.4-2.8$ \\
\hline 49.62 & Purple & 29.8 & 0.72 & 8.2 & 2.7 & 4 & 2.3 & $10.3-10.5$ & $1.5-2.0$ & $1.9-2.2$ & $37.47-37.62$ & $0.27-0.37$ & $4.4-4.7$ \\
\hline
\end{tabular}

Notes.

a Day after discovery, 2008 April 10.7.

b Net exposure time.

c $10^{-10}$ erg cm $\mathrm{cm}^{-2} \mathrm{~s}^{-1}$ over range $11-37 \AA$

d From blackbody fit.

e $10^{21} \mathrm{~cm}^{-2}$.

f Oxygen abundance in TBabs model, relative to solar by Grevesse \& Sauval (1998).

g From atmosphere model.

While the OM light curve appears to show some anticorrelated variations to the X-ray light curves, these cannot be considered significant as the OM light curve is not variable on a statistically significant level. When ignoring the measurement errors, a Spearman rank test yields no correlation or anticorrelation between the X-ray and UV light curves.

During the second observation, the X-ray brightness (binned in $50 \mathrm{~s}$ bins) deviates only marginally from a constant rate, yielding $\chi_{v}^{2} \sim 2.9$ with 594 degrees of freedom. For the OM light curves, binned on $50 \mathrm{~s}$ grids, the assumption of constant light curves yields $\chi_{v}^{2}$ of 1.2 for the UVW2 light curve (which has the lowest average count rate), 1.6 for the two UVM2 light curves, but 4.2 and 4.6 for the best-exposed UVW1 light curves. All fits have been done with 42 degrees of freedom.

The higher degree of variability in X-rays during the first observation, especially with the deep dip, is consistent with a high degree of variations in the early Swift/XRT light curve (see Figure 1), while the second observation was taken at a time when the Swift X-ray light curve was also stabler with no dips (Page et al. 2010). Meanwhile, the long-term UV light curve taken with the UVOT on board Swift shows a slow, continuous decay with no signs of variability (see the middle panel of Figure 1). The XMM-Newton light curves fit into this general picture with a high degree of variability in X-rays and a lower degree of variability in the UV.

\subsection{Timing Analysis}

We have performed period studies using the EPIC/pn, EPIC/ MOS, and RGS light curves of the first observation, all giving consistent results. As discussed above, the OM light curves and the second observation are not significantly variable for period studies. In order to remove long-term trends, we have detrended the light curves with an eighth-order polynomial and applied a Lomb-Scargle algorithm (Scargle 1982) and a 2-sine fitting method developed by Dobrotka \& Ness (2010). 99.7\% errors are calculated from the false alarm probability (Horne $\&$ Baliunas 1986) for the Lomb-Scargle analysis and from $3 \sigma$ confidence isocontours for the 2 -sine fitting method. Since no variability is present during the dip, we have excluded the data between 8 and $22 \mathrm{ks}$ of elapsed time.

The results from the Lomb-Scargle procedure are shown in Figure 4, derived from the detrended light curves from EPIC/MOS (black) and EPIC/pn (blue). In the top panel, the initial result is shown with two significant frequencies of $f_{1}=0.440 \pm 0.033 \mathrm{mHz}$ and $f_{2}=0.465 \pm 0.035 \mathrm{mHz}$.

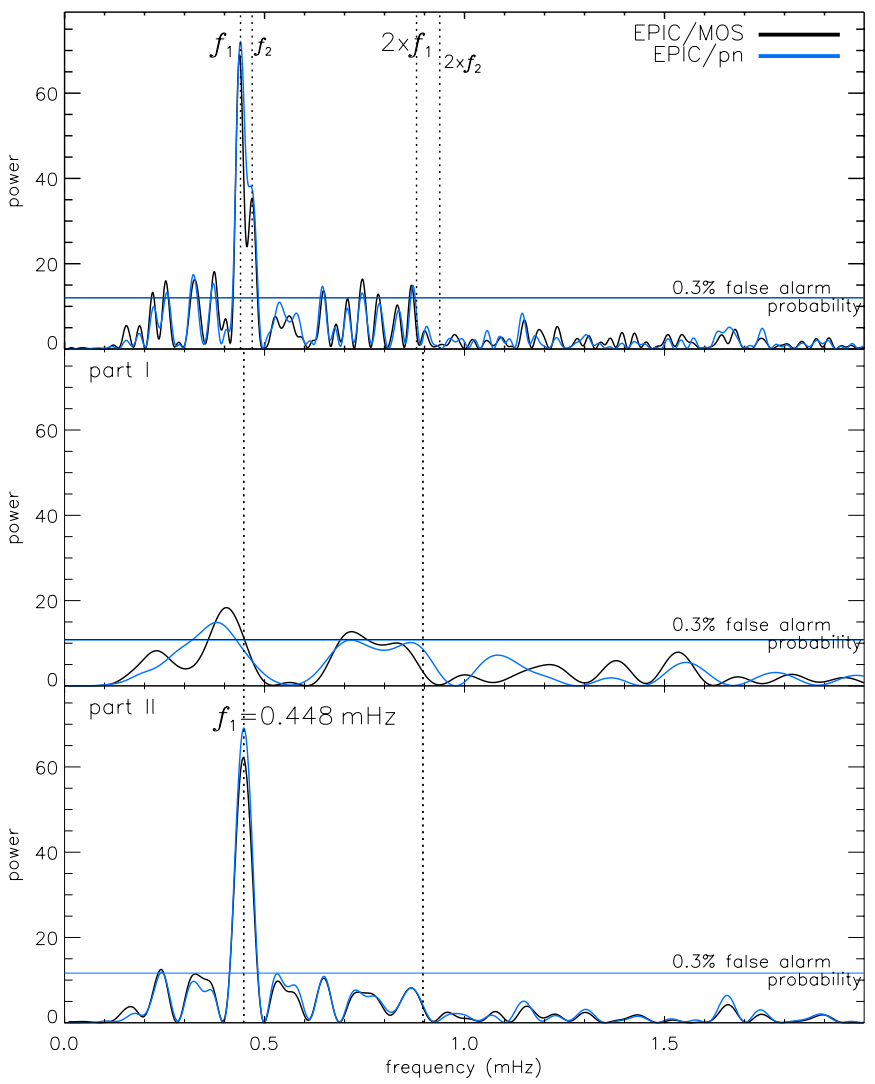

Figure 4. Results from Lomb-Scargle period analysis applied to the EPIC/ MOS (black) and EPIC/pn (blue) detrended light curves while excluding the time interval between 8 and $22 \mathrm{ks}$ of elapsed time to avoid contamination by the dip. A false alarm probability level of $0.3 \%$ is marked by the horizontal line, and all peaks above this line can be considered statistically significant at the 99.7\% $(3 \sigma)$ level. Two nearby significant frequency values $f_{1}$ and $f_{2}$ are marked in the top panel, together with the expected frequencies of their first harmonics. Separate periodograms calculated for the first $8 \mathrm{ks}$ of data before the dip (part I) and those after $22 \mathrm{ks}$ (part II) have been computed and are shown in the bottom two panels.

(A color version of this figure is available in the online journal.)

The expected location of the first harmonic to each detected frequency is marked by a vertical dotted line, and at most marginal evidence for harmonics is present.

In addition, we have calculated two separate periodograms for two parts. Part I represents the first $8 \mathrm{ks}$ before the dip and part II ( $22 \mathrm{ks}$ after the dip). The results are shown in the bottom 


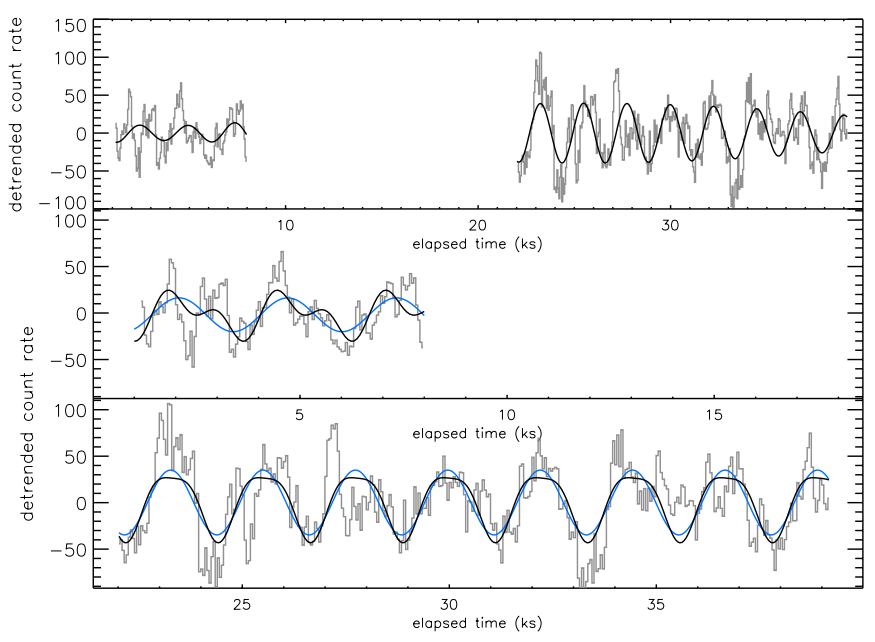

Figure 5. Top panel: result from fitting two sine curves to the detrended EPIC/ pn light curve. The data are in light gray and model in black. The strongest peak in the Lomb-Scargle periodograms are shown in the last two panels in Figure 4 and as sine curves overplotted over the first part (middle) and the second part (bottom). The blue curves are single frequencies of $0.381 \mathrm{mHz}$ and $0.448 \mathrm{mHz}$, and the black curves are the sum of the ground frequency and the respective first harmonic.

(A color version of this figure is available in the online journal.)

two panels of Figure 4. While the first part is too short for any significant detection, the second, longer, part clearly yields only a single frequency of $0.448 \pm 0.036 \mathrm{mHz}$, corresponding to a period of 37.2 minutes.

In order to investigate why the total light curve yields two significant frequencies, we have performed several tests. We applied the sine fitting method by Dobrotka \& Ness (2010) and found two frequencies. However, a synthetic light curve modulated with a single frequency, sampled and noised as the $\mathrm{EPIC} / \mathrm{pn}$ data, also yields two frequencies. We identify the reason to be an increase in the amplitude of variations after the dip, which mimics a beating cycle when fitted with two sine curves. This is illustrated in Figure 5 where the detrended EPIC/ pn light curve is shown in gray with the best-fit 2-sine curve overplotted. One can clearly see that the changes in amplitude in the model reproduce the amplitude change in the data. While the change in amplitude could be beating of two signals, we consider it more likely that some other process that is related to the dip is responsible for the change in amplitude.

For the discussion of the origin of the oscillations it is important to know whether the first harmonic is also present. In the bottom panels of Figure 5, we show the first and second parts with two different light curve fits based on the frequencies detected in the respective periodograms (see bottom two panels of Figure 4). The light blue lines are single sine curves which were obtained after fitting amplitudes and phase while keeping the frequencies fixed at $0.381 \mathrm{mHz}$ for the first part and $0.448 \mathrm{mHz}$ for the second part. The black curves are fits with fixed fundamental frequencies plus the respective first harmonic (twice the frequency values), also with variable phase shifts and amplitudes. While the detection of a first harmonic is in no case statistically significant (as can be seen from Figure 4), the best fit to the first part suggests that it may be present but is not significant only for the reason that no more than three cycles are covered. We can therefore not fully exclude that a first harmonic was present before the dip which then disappeared in the dip, as is clearly not present in the second part.
The frequency at $0.448 \pm 0.036 \mathrm{mHz}$ (with a pulse fraction of $7 \%$ after the dip) seems clearly detected, but we wish to caution that only seven cycles are covered, bearing a certain risk of red noise. This has been discussed in detail by Vaughan (2010), and references therein. We tested the confidence of our signal using the method proposed by Vaughan (2005). The $0.448 \mathrm{mHz}$ peak is approximately at the $98 \%$ confidence limit. More data could mitigate this situation, but the entire observation cannot be used because the dip may have changed the conditions under which a periodic signal is visible. The second observation yields no periodic signal. Our conclusions are based on seven apparently periodic cycles in the second part of the first observation.

\section{SPECTRA}

The SAS tool rgsproc was used to generate RGS events files from the raw data and extract spectra in $0.01 \AA$ bins from a standardized extraction region around the dispersed photons. Time filters can be applied, allowing for the accumulation of spectra over any given time interval. The SAS tool rgsfluxer combines all information from both spectrometers into a single spectrum in photon flux units. It does not correct for redistribution of monochromatic response into the dispersion channels. Thus, the "fluxed" spectrum is not suitable for quantitative analyses but it can be used for the purpose of visualization and comparison with broadband models or other observations.

\subsection{Description}

In Figure 6, the fluxed RGS spectra from four different epochs are shown. The color code is the same as that used in Figure 3. In Table 3, the respective start times in units of day after discovery, exposure time, integrated X-ray band fluxes, and best-fit blackbody parameters (see below) are listed. The fluxes are absorbed fluxes and correspond to logarithmic luminosities of $30.67,30.11,30.82$, and 29.98, respectively (in cgs units). The spectra are all continuous spectra with deep absorption lines at, e.g., $\sim 19 \AA$ and $\sim 24.5 \AA$. The overall shape of the spectra resembles that of an absorbed blackbody, which is demonstrated by the overplotted best-fit blackbody curves that were obtained by $\chi^{2}$ minimization. Spectral bins around deep absorption lines have been discarded for better reproduction of the continuum. The effects of photoelectric absorption along the line of sight were modeled using the Boulder-Tübingen model TBabs developed by Wilms et al. (2000) with variable oxygen abundance. The best-fit parameters are listed in the last four columns of Table 3, where the last column lists the oxygen abundance in the TBabs model, relative to solar (Grevesse \& Sauval 1998). It was allowed to vary in order to reproduce the depth of the O I absorption edge at $\sim 23 \AA$. The corresponding $1 s-2 p$ absorption line at $23.5 \AA$ is also modeled as part of TBabs. The other absorption lines are of photospheric origin, indicating that the spectrum is that of an atmosphere. The deepest photospheric absorption lines at $\sim 19$ and $\sim 24.5 \AA$ have been excluded from the fit.

With the given complex structure of the expanding nova ejecta, the blackbody fits are only parameterizations. As shall be shown in Section 4.4, obtaining reliable results from atmosphere modeling is sufficiently complex to require a separate project, and the blackbody temperature is here given only as a preliminary characterization of the spectral hardness. The statistical uncertainties are small, of order $3 \%$, because of the high quality of the data, however, the values of reduced $\chi^{2}$ are 2.4, 1.8, 8.4, and 2.0 for days $39.93,40.03,40.18$, and 49.62, respectively, 


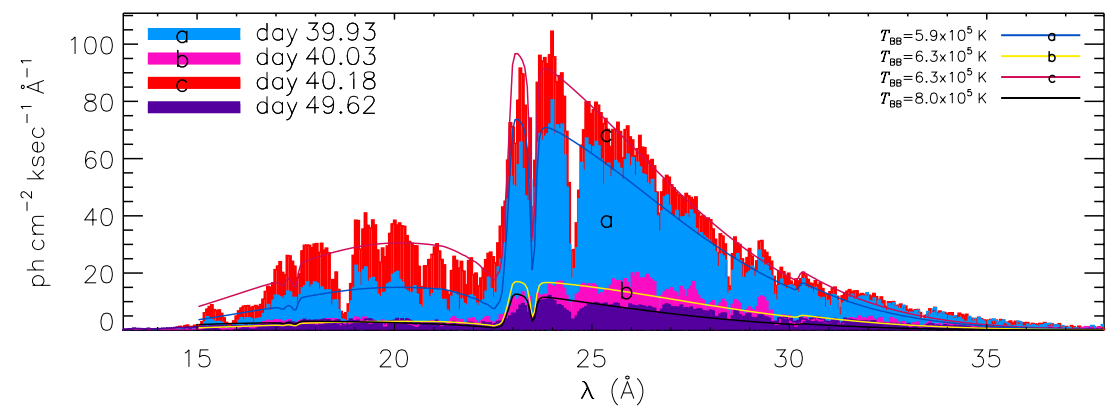

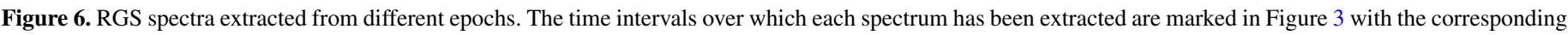

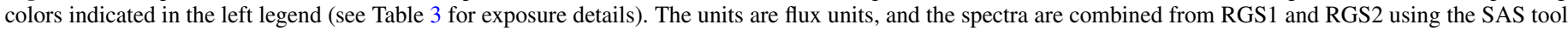
rgsfluxer. The thin lines are best-fit blackbody fits with the blackbody temperatures given in the right legend.

(A color version of this figure is available in the online journal.)

and standard error determination is not reliable for large values of reduced $\chi^{2}$. Blackbody fits without discarding any spectral bins yield systematically higher temperatures by $\sim 0.5 \times 10^{5} \mathrm{~K}$, with much larger values of reduced $\chi^{2}$ of $6.3,2.2,28.8$, and 3.5. We emphasize that these temperatures cannot be interpreted as the photospheric temperature because a blackbody model does not account for the most fundamental physics in the ejecta.

For comparison with the observed UV fluxes listed in Table 2, we have calculated the projected fluxes from the blackbody fits. For days 39.93, 40.03, 40.18, and 49.62, we found $(0.61,1.5$, and 2.1$) \times 10^{-14} \mathrm{erg} \mathrm{cm}^{-2} \mathrm{~s}^{-1} \AA^{-1},(0.06$, 0.15 , and 0.21$) \times 10^{-14} \mathrm{erg} \mathrm{cm}^{-2} \mathrm{~s}^{-1} \AA^{-1},(0.43,1.1$, and 1.5) $\times 10^{-14} \mathrm{erg} \mathrm{cm}^{-2} \mathrm{~s}^{-1} \AA^{-1}$, and $(1.9,4.9$, and 6.9) $\times$ $10^{-17} \mathrm{erg} \mathrm{cm}^{-2} \mathrm{~s}^{-1} \AA^{-1}$ for the UVW1, UVM2, and UVW2 filters, respectively. These fluxes are significantly lower than the measured values. Since other sources of UV emission are likely present, these numbers do not rule out a blackbody nature of the broadband spectrum.

The changes in blackbody temperature are fairly small between the three epochs of the first observation, which is consistent with the findings by Page et al. (2010). However, the second observation yields a significantly harder spectrum. At the same time, the best-fit value of $N_{\mathrm{H}}$ is of the same order as the interstellar value derived from $E(B-V)$ measurements, indicating that the previous observations contained excess absorption from local neutral material. Note also that Page et al. (2010) found even lower values of $N_{\mathrm{H}}$ around day 50 (see their Figure 4). While the increases in spectral hardness could be due to an increasing effective temperature, which would be consistent with a smaller effective blackbody radius, changes in the atmospheric structure may also have occurred. Also, an increasing degree of ionization of circumbinary neutral material can lead to spectral hardening as demonstrated by Ness et al. (2007) for RS Oph. The degree of ionization of oxygen influences the depth of the O I absorption edge at $23 \AA$ and the associated $1 s-2 p$ line at $23.5 \AA$. The parameter $A(\mathrm{O})$, the abundance of neutral oxygen in the cold absorber component, yields higher values for a lower degree of ionization because more neutral oxygen is present. The results given in the last column of Table 3 indicate that on day 40.03, when the total flux was lower, the degree of ionization was also lower, which can be explained by recombination in a photoionized plasma that was less irradiated during the times of low-flux emission.

Some model-independent conclusions can be drawn by direct inspection of the details in the spectra. Trends of abundances can be estimated from line depths, and the local expansion velocity can be determined from line shifts on a quantitative level.

\subsection{Details in the High-resolution Spectra}

The details of the spectra are shown in Figure 7, using the same color code as in Figures 3 and 6. Line labels of prominent transitions are placed at their rest wavelengths using different colors that indicate groups of transitions and elements. The groups are neutral (interstellar) lines (black), the H-like Ly series $(1 s-n p)$ of $\mathrm{N}$ VII, O VIII, and C VI (black, red, and blue, respectively), the He-like $1 s-n p$ series of $\mathrm{N}$ VI, $\mathrm{O}$ VII, and $\mathrm{C} \mathrm{V}$ (purple, orange, and gray), Neon emission lines (cyan), and absorption features that cannot be identified (green). Lines that are not detected but are of interest are marked in light gray (e.g., $\mathrm{C} \mathrm{v}$ ). Expected blueshifts corresponding to a Doppler velocity of $-3200 \mathrm{~km} \mathrm{~s}^{-1}$ are indicated by dotted lines for each transition. For the majority of lines, the dotted lines coincide with deep absorption troughs. Except for the two interstellar lines of O I $(23.5 \AA)$ and $N_{I}(31.28 \AA)$, all lines indicate roughly the same expansion velocity of $-3200 \mathrm{~km} \mathrm{~s}^{-1}$.

In the spectrum representing the low emission phase during the first observation (phase $b$, pink), the N vII and the C VI Ly $\alpha$ lines at $\lambda_{0}=24.78 \AA$ and $\lambda_{0}=33.74 \AA$, respectively, appear to be blueshifted by a larger amount than in the other two spectra from the first observation (phases a and $\mathrm{c}$ in blue and red). Meanwhile, the $\operatorname{Ly} \beta$ lines of the same elements and the N VI lines show the same blueshift.

The H-like Ly series lines of N viI can be identified up to $1 s-5 p(\mathrm{Ly} \delta)$. The recombination/ionization continuum of $\mathrm{N}$ VII is expected at $18.59 \AA(667.1 \mathrm{eV})$ but is not seen (and is not marked) because it overlaps with the O vII $1 s-3 p$ line at $18.67 \AA$. For O vIII, the $1 s-n p$ series can be seen up to $n=4(\operatorname{Ly} \gamma)$. At shorter wavelengths, not enough continuum is present to detect higher- $n$ Ly series lines of O VIII. For C VI, the $1 s-n p$ series can only be seen up to $\operatorname{Ly} \gamma$, while $\operatorname{Ly} \delta$ and the recombination/ ionization continuum at $25.3 \AA(490 \mathrm{eV})$ are not present. The Helike series lines of $\mathrm{N}$ VI and $\mathrm{O}$ VII are detected up to $1 s-5 p$, and for $\mathrm{O}$ VII, the recombination/ionization continuum at $16.77 \AA$ $(739.3 \mathrm{eV})$ is present between 16.6 and $16.8 \AA$. This is shown in more detail in Figure 8 . The depth of the line is strongest in the spectrum from day 40.18. The shape of the edge indicates an imbalance in favor of ionization, thus $\mathrm{O}$ VIII is being pumped by photoionization. The $\mathrm{N}$ VI recombination/ionization continuum at $22.46 \AA(552.1 \mathrm{eV})$ cannot be detected because it overlaps with a number of low-ionization oxygen lines, $\mathrm{O} v \mathrm{v}, \mathrm{O} v$, and O IV, marked in red. While the He-like $1 s-2 p$ line of $\mathrm{CV}$ $(40.27 \AA$ ) is outside the spectral range of the RGS, the highorder series lines are in the RGS bandpass and are not detected (gray labels). 


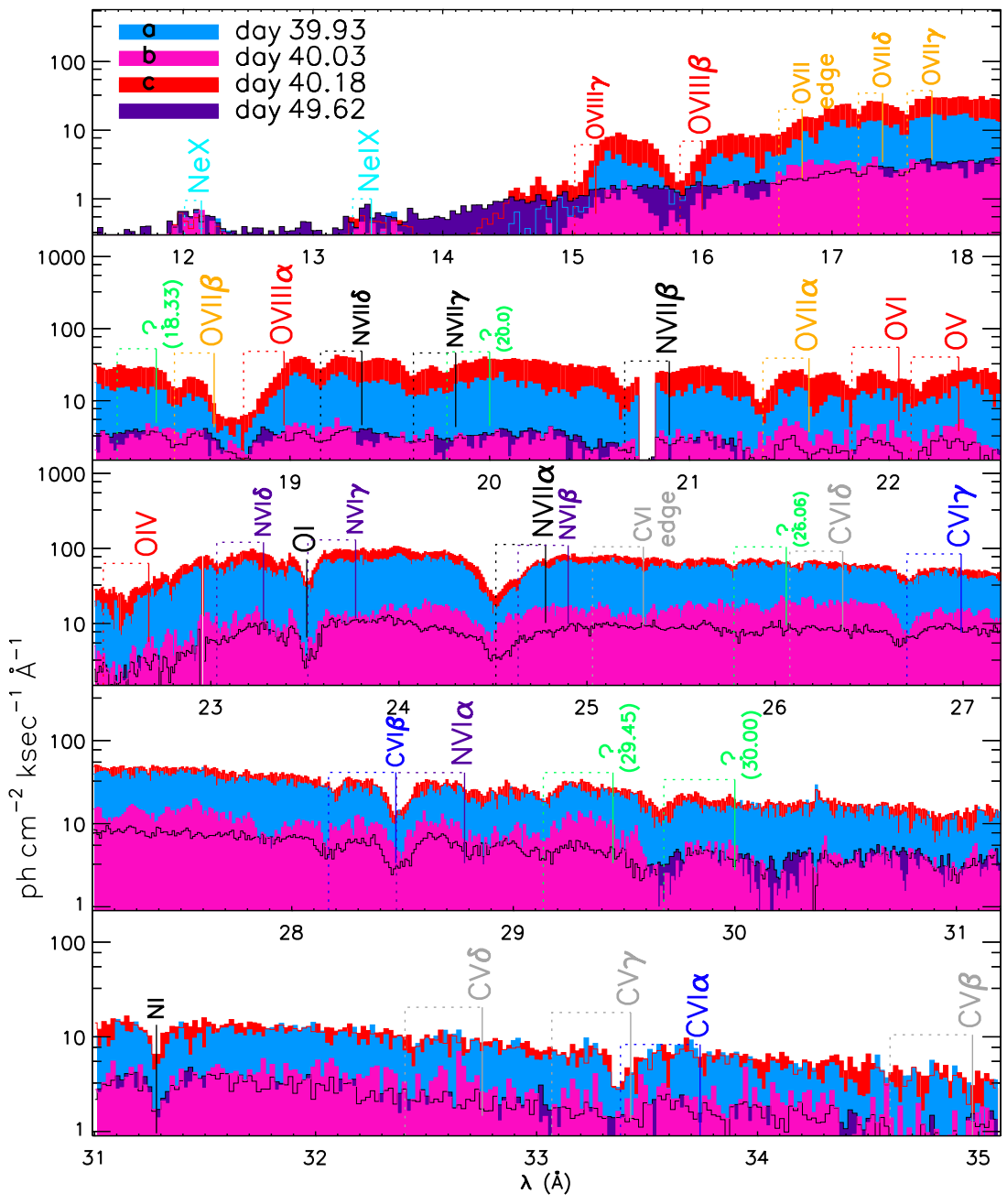

Figure 7. Combined "fluxed" RGS1 and RGS2 spectra of V2491 Cyg extracted from different time intervals. The start times are given in the upper left legend, and the colors correspond to those used in Figure 3 (see Table 3). Important absorption lines are marked with small vertical solid lines at their rest wavelengths and with dashed lines at the wavelengths corresponding to blueshifts of $v=-3200 \mathrm{~km} \mathrm{~s}^{-1}$. The line labels are color coded indicating lines of $\mathrm{N}$ VII in black, $\mathrm{N}$ VI in purple, O IV-VI and $\mathrm{O}$ VIII in red, O VII in orange, carbon lines in blue, interstellar lines in black, and unidentified lines in green. Labels in gray indicate lines that are not detected.

(A color version of this figure is available in the online journal.)

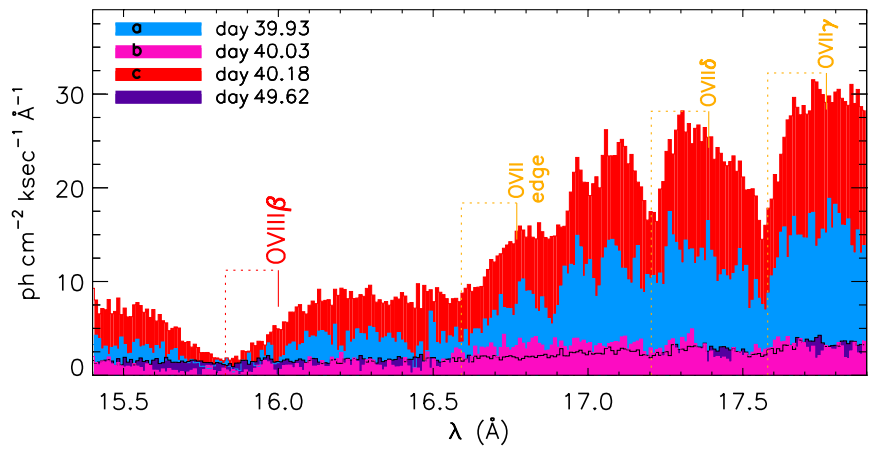

Figure 8. Same as Figure 7 in linear units, focusing on the O vir edge at $16.77 \AA$. (A color version of this figure is available in the online journal.)

Unidentified absorption features are marked with a question mark at a wavelength that would be the rest wavelength if that feature was photospheric and thus blueshifted by the same amount as the other lines, but they could also be interstellar lines. Identification of these lines might be possible if they were also seen in other nova spectra with a different velocity profile (see Section 5.2.2). Seeing similar features at the same wavelengths would be an argument in favor of interstellar lines, while line shifts by an amount commensurate with other line shifts would be more suggestive of photospheric lines.

At 12 and $13.5 \AA$, the H-like and He-like lines of $\mathrm{NeX}$ and Ne IX are clearly detected in emission. On day 49.62, this wavelength region is dominated by continuum emission originating from an increased Wien tail of the photospheric continuum spectrum. In the logarithmic plot it is difficult to see, but the Ne lines are still significantly present, albeit with a lower flux (Ness et al. 2008b).

\subsection{Line Profile Modeling}

The deepest absorption line is the Ly $\alpha$ line of O VIII at $18.8 \AA$ $\left(\lambda_{0}=18.97 \AA\right)$. Ness (2010) found that it was saturated without going to zero. This is the only line for which the bottom of the line profile is flat, indicating that it has the largest column density and thus the highest number density. In addition to a high oxygen abundance, the temperature structure at the time of the observation provides particularly advantageous conditions for the formation of the $\mathrm{O}$ VIII ionization state. The latter is supported by the presence of the $\mathrm{O}$ VII to $\mathrm{O}$ VIII ionization edge at $16.8 \AA$ (see Figure 8), which is an indicator for photoionization. 
Table 4

Results from Line Profile Measurements

\begin{tabular}{|c|c|c|c|c|c|c|c|c|c|c|c|}
\hline \multicolumn{2}{|c|}{$\begin{array}{l}\operatorname{Ion} \log (\xi) \\
\left(\mathrm{erg} \mathrm{s}^{-1} \mathrm{~cm}\right)\end{array}$} & $\begin{array}{l}\lambda_{0} \\
(\AA)\end{array}$ & $f$ & $\begin{array}{c}v_{\text {shift }} \\
\left(\mathrm{km} \mathrm{s}^{-1}\right)\end{array}$ & $\begin{array}{c}v_{\text {width }} \\
\left(\mathrm{km} \mathrm{s}^{-1}\right)\end{array}$ & $\tau_{\mathrm{c}}$ & $\begin{array}{c}N_{\mathrm{X}} \\
\left(10^{16} \mathrm{~cm}^{-2}\right)\end{array}$ & $\begin{array}{c}v_{\text {shift }} \\
\left(\mathrm{km} \mathrm{s}^{-1}\right)\end{array}$ & $\begin{array}{c}v_{\text {width }} \\
\left(\mathrm{km} \mathrm{s}^{-1}\right)\end{array}$ & $\tau_{\mathrm{c}}$ & $\begin{array}{c}N_{\mathrm{X}} \\
\left(10^{16} \mathrm{~cm}^{-2}\right)\end{array}$ \\
\hline \multicolumn{8}{|c|}{ Day 39.93} & \multicolumn{4}{|c|}{ Day 40.18} \\
\hline $\mathrm{C} \mathrm{VI} \beta$ & 1.30 & 28.46 & 0.16 & $-2705_{-172}^{+146}$ & $<353$ & $0.37_{-0.35}^{+51.01}$ & $<1.41$ & $-3034_{-135}^{+131}$ & $687_{-213}^{+280}$ & $0.29 \pm 0.09$ & $0.27 \pm 0.09$ \\
\hline $\mathrm{C}$ VI $\alpha$ & 1.30 & 33.74 & 0.83 & $-3010_{-220}^{+230}$ & $626_{-197}^{+313}$ & $0.94_{-0.37}^{+0.57}$ & $0.12_{-0.05}^{+0.08}$ & $-3213_{-90}^{+113}$ & $<549$ & $0.90_{-0.24}^{+0.35}$ & $0.12_{-0.03}^{+0.05}$ \\
\hline $\mathrm{N}$ VI $\beta$ & 0.80 & 24.90 & 0.14 & $-3157_{-213}^{+140}$ & $567_{-191}^{+292}$ & $0.46 \pm 0.15$ & $0.62 \pm 0.21$ & $(-3041 \pm 57)^{\mathrm{a}}$ & $\left(726_{-72}^{+79}\right)^{\mathrm{a}}$ & $(0.43 \pm 0.03)^{\mathrm{a}}$ & $(0.57 \pm 0.05)^{\mathrm{a}}$ \\
\hline $\mathrm{N}$ VI $\alpha$ & 0.80 & 28.78 & 0.66 & $-3119_{-87}^{+94}$ & $631_{-116}^{+150}$ & $0.87 \pm 0.20$ & $0.19 \pm 0.05$ & $-3147 \pm 40$ & $611_{-54}^{+58}$ & $0.90 \pm 0.09$ & $0.20 \pm 0.02$ \\
\hline N VII $\alpha$ & 1.60 & 24.78 & 0.83 & $-3275_{-143}^{+107}$ & $1146_{-139}^{+144}$ & $1.30 \pm 0.10$ & $0.29 \pm 0.03$ & $(-3229 \pm 27)^{\mathrm{a}}$ & $\left(1108_{-28}^{+29}\right)^{\mathrm{a}}$ & $(1.12 \pm 0.03)^{\mathrm{a}}$ & $(0.25 \pm 0.01)^{\mathrm{a}}$ \\
\hline $\mathrm{O}$ VII $\beta$ & 1.20 & 18.63 & 0.15 & $-3227_{-136}^{+150}$ & $777_{-187}^{+263}$ & $0.78 \pm 0.31$ & $1.84 \pm 0.82$ & $-3178 \pm 58$ & $965_{-90}^{+95}$ & $0.91 \pm 0.08$ & $2.08 \pm 0.23$ \\
\hline O VII $\alpha$ & 1.20 & 21.60 & 0.68 & $-3175_{-193}^{+186}$ & $716_{-200}^{+290}$ & $0.81_{-0.25}^{+0.34}$ & $0.31_{-0.11}^{+0.14}$ & $-3308 \pm 64$ & $855_{-87}^{+100}$ & $0.95 \pm 0.11$ & $0.35 \pm 0.05$ \\
\hline $\mathrm{O}$ vIII $\beta$ & 2.70 & 16.00 & 0.16 & $-3292_{-324}^{+319}$ & $2752_{-493}^{+613}$ & $1.30 \pm 0.29$ & $3.47 \pm 1.08$ & $-3275 \pm 107$ & $2825_{-188}^{+205}$ & $1.41 \pm 0.09$ & $3.52 \pm 0.36$ \\
\hline O VIII $\alpha$ & 2.70 & 18.97 & 0.83 & $-3703 \pm 107$ & $2545_{-283}^{+361}$ & $2.37 \pm 1.25$ & $0.84_{-0.41}^{+0.53}$ & $-3732 \pm 41$ & $2302_{-97}^{+111}$ & $3.24 \pm 0.52$ & $1.13 \pm 0.22$ \\
\hline \multicolumn{8}{|c|}{ Day 40.03} & \multicolumn{4}{|c|}{ Day 49.62} \\
\hline $\mathrm{C} \mathrm{VI} \beta$ & 1.30 & 28.46 & 0.16 & $-3235_{-174}^{+237}$ & $<487$ & $0.54_{-0.46}^{+5.29}$ & $<7.23$ & $-2996_{-192}^{+201}$ & $558_{-178}^{+226}$ & $0.36_{-0.14}^{+0.18}$ & $0.33_{-0.14}^{+0.18}$ \\
\hline $\mathrm{C}$ VI $\alpha$ & 1.30 & 33.74 & 0.83 & $-3603_{-155}^{+236}$ & $<561$ & $1.57_{-0.59}^{+34.13}$ & $<1.03$ & $\ldots$ & $\ldots$ & $\ldots$ & $\ldots$ \\
\hline $\mathrm{N}$ VI $\beta$ & 0.80 & 24.90 & 0.14 & $-3044_{-316}^{+293}$ & $398_{-398}^{+553}$ & $0.23_{-0.15}^{+0.69}$ & $0.31_{-0.21}^{+1.17}$ & $-2516_{-210}^{+205}$ & $<920$ & $0.19_{-0.13}^{+0.16}$ & $0.25_{-0.18}^{+0.25}$ \\
\hline $\mathrm{N}$ VI $\alpha$ & 0.80 & 28.78 & 0.66 & $-3106 \pm 129$ & $364_{-364}^{+194}$ & $0.72_{-0.26}^{+0.39}$ & $0.16_{-0.06}^{+0.10}$ & $-3165 \pm 76$ & $656_{-85}^{+101}$ & $0.82 \pm 0.15$ & $0.18 \pm 0.04$ \\
\hline N vII $\alpha$ & 1.60 & 24.78 & 0.83 & $-3661_{-144}^{+170}$ & $1230_{-254}^{+349}$ & $0.76 \pm 0.14$ & $0.17 \pm 0.04$ & $-3029 \pm 85$ & $1150_{-143}^{+176}$ & $0.79 \pm 0.09$ & $0.17 \pm 0.03$ \\
\hline $\mathrm{O}$ VII $\beta$ & 1.20 & 18.63 & 0.15 & $-2844_{-583}^{+357}$ & $<7903$ & $0.59_{-0.40}^{+10.63}$ & $<22.10$ & $-3053 \pm 144$ & $766_{-156}^{+206}$ & $1.19_{-0.34}^{+0.50}$ & $2.84_{-0.91}^{+1.38}$ \\
\hline O vII $\alpha$ & 1.20 & 21.60 & 0.68 & $\ldots$ & $\ldots$ & $\ldots$ & $\ldots$ & $-3327 \pm 174$ & $777_{-202}^{+249}$ & $0.79 \pm 0.29$ & $<0.43$ \\
\hline $\mathrm{O}$ VIII $\beta$ & 2.70 & 16.00 & 0.16 & $-3964_{-544}^{+536}$ & $4263_{-878}^{+1296}$ & $1.43 \pm 0.36$ & $3.81 \pm 1.34$ & $-3137_{-477}^{+422}$ & $894_{-586}^{+711}$ & $0.28_{-0.15}^{+0.33}$ & $0.82_{-0.49}^{+1.08}$ \\
\hline $\mathrm{O}$ vIII $\alpha$ & 2.70 & 18.97 & 0.83 & $-3721_{-337}^{+316}$ & $2360_{-461}^{+608}$ & $2.16_{-0.63}^{+1.20}$ & $0.81_{-0.29}^{+0.55}$ & $-3776 \pm 102$ & $908_{-167}^{+163}$ & $5.85_{-2.32}^{+9.17}$ & $<13.06$ \\
\hline
\end{tabular}

Note. ${ }^{\text {a }}$ Uncorrelated errors given, see the text for details.

We have used a slightly modified version of the method described by Ness (2010) to determine line shifts, widths, and optical depths at line center for each of the four spectra from days $39.93,40.03,40.18$, and 49.62. A line profile model spectrum is calculated over a narrow wavelength range around an absorption line, based on Equation (1) in Ness (2010). For faster computation, we perform no correction for interstellar absorption (thus $T(\lambda) \equiv 1$ ), and for the continuum we assume a linear function rather than a blackbody curve. These two effects are marginal over the small wavelength region around the absorption lines. We have compared the results by Ness (2010) with the same spectra and lines but with the modified method and get consistent results.

The resulting parameters of interest are the optical depth at the line center, $\tau_{\mathrm{c}}$, the central (rest) wavelength, $\lambda_{0}$, and the Gaussian line width, $\sigma$. The line shifts, $\left(\lambda-\lambda_{0}\right)$, and line widths were converted to Doppler velocities $v_{\text {shift }}=c\left(\lambda-\lambda_{0}\right) / \lambda_{0}$ and $v_{\text {width }}=c \sigma / \lambda_{0}$, where $c$ is the speed of light. The instrumental line broadening can be estimated as $v_{\text {instr }} \sim 300 \mathrm{~km} \mathrm{~s}^{-1}$ and was accounted for in computing $v_{\text {width }}=\sqrt{\left(c \sigma / \lambda_{0}\right)^{2}-v_{\text {instr }}^{2}}$.

In addition to these parameters, line column densities, $N_{\mathrm{X}}$, were obtained by integration over the opacity $\tau(\lambda)$ as defined in Equation (2) of Ness (2010), using oscillator strengths extracted from the Chianti database (Landi et al. 2006), which is an approximation for strong lines.

All results are summarized in Table 4, where the top block lists the results for the two brightest spectra taken before and after the dip in the first observation (blue and red shadings in the top panel of Figure 3), and the bottom block represents the two faintest spectra represented by pink and purple colors in Figure 7. Given are the name of ion, ionization parameter (see last paragraph of this subsection), rest wavelength, oscillator strength, line shift and width, optical depth at the line center, and line column density. The given errors were obtained for each parameter from a grid of parameter values versus corresponding $\chi^{2}$ by interpolating the two parameter values on each side of the minimum that are larger by 3.53 than the minimum $\chi^{2}$. This increase in $\chi^{2}$, with three free parameters of interest, yields $1 \sigma$ uncertainties. While stepping through the grid, all other parameters were iterated, including the parameters for the continuum that are statistically "uninteresting" (see Avni 1976), starting at their best-fit values. If a better fit was found during this procedure, a new minimization was started with the new parameters until the fit was stable. With this procedure, we are confident to have found global minima in $\chi^{2}$. Only in one case have we not accepted the best fit, which is illustrated in Figure 9, where in the top panel the global best fit is given while in the bottom panel our preferred fit is shown, which is a local minimum in $\chi^{2}$. The best fit requires a broad N VI line and a narrow N VII line, while all other observations yield the opposite result. This single result does not agree with the other results. We illustrate this to make the point that rigorous fitting does not always lead to the best results. In Figure 9, a dip between -2000 and $-1800 \mathrm{~km} \mathrm{~s}^{-1}$ can be seen which could well be originating from the $\mathrm{N}$ VI transition. In the bottom panel, this dip is much better reproduced, yielding a velocity shift for $\mathrm{N}$ vi that is consistent with that of the large majority of other absorption lines. Although this fit has a higher value of $\chi^{2}$, we prefer this fit over the one in the top panel, and in Table 4, the best-fit values are given.

Figure 10 is a graphical illustration of the line shifts and line widths (top panel) for different lines at different times. Only strong $1 s-2 p$ (Ly $\alpha$ and $\operatorname{He} \alpha$ ) transitions were selected from Table 4 for this plot for better clarity. The majority of lines are blueshifted by -3000 to $-3400 \mathrm{~km} \mathrm{~s}^{-1}$ and are broadened by up to $1200 \mathrm{~km} \mathrm{~s}^{-1}$, which is significantly broader than the expected 


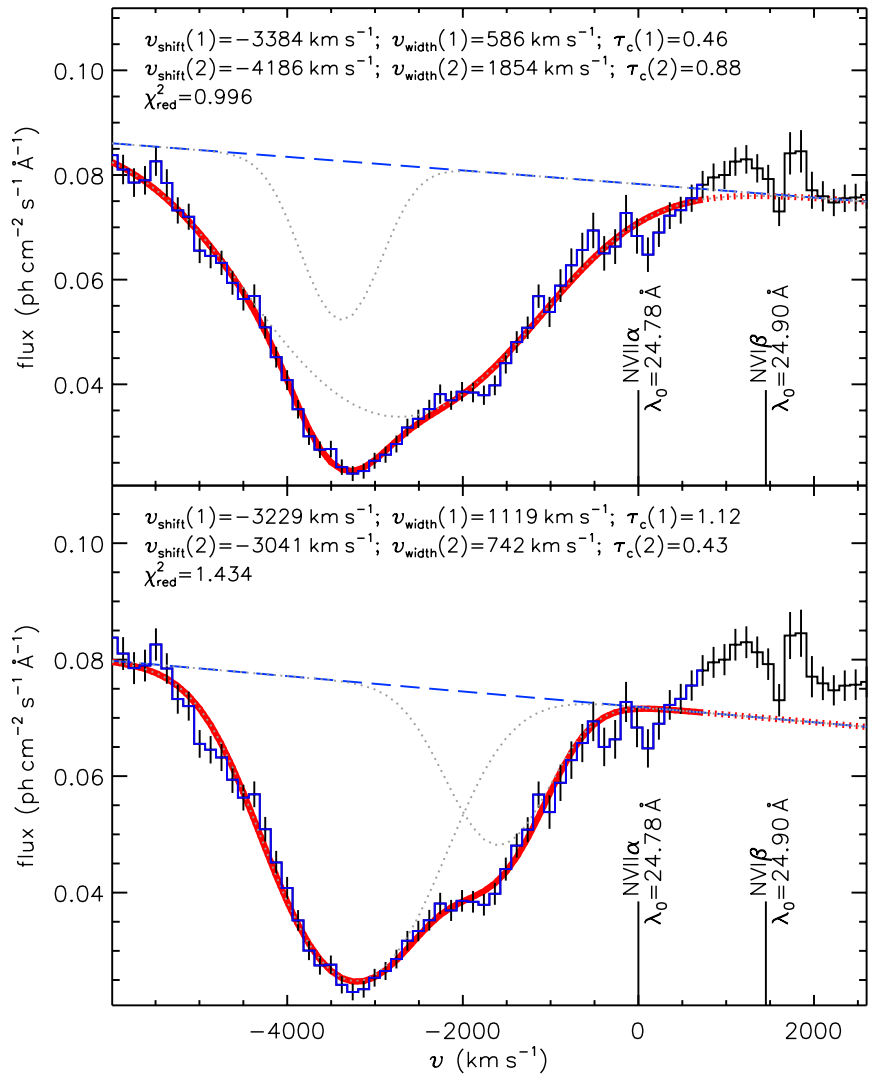

Figure 9. Line profile fitting to the spectrum of day 40.18, following a modified method by Ness (2010, see the text) for the two blended lines of N VII $\left(\lambda_{0}=24.78 \AA\right)$ and N VI $\left(\lambda_{0}=24.90 \AA\right)$. The observed spectrum is plotted in histogram style (blue for fitted part and black for non-fitted part), the best-fit model with a thick red line (solid and dotted for fitted and non-fitted, respectively), and the model components for each line with gray dotted lines. While the fit in the top panel yields lower $\chi^{2}$, we consider the fit in the bottom panel as the one with better physical motivation because the parameters are closer to the values from the other spectra (see Table 4), and a dip between -2000 and $-1800 \mathrm{~km} \mathrm{~s}^{-1}$ is better reproduced.

(A color version of this figure is available in the online journal.)

line width from instrumental broadening. We can thus conclude that the ejecta are significantly extended, allowing us to view a range of expansion velocities and thus a range of different plasma layers.

The $\mathrm{O}$ VIII line clearly stands out with consistently larger line shifts and widths in all three data sets of the first observation. By day 49.6, the line has become narrower but has the same line shift.

The N VII and C VI lines are blueshifted by a larger amount during the dip in the first observation (day 40.03 in pink). Together with the $\mathrm{O}$ VIII line, these are all $\mathrm{H}$-like transitions while the He-like $\mathrm{N}$ vi line, which is formed at lower temperatures, is not more blueshifted during the dip. This could indicate that the high-temperature lines probe higher wind velocities which are likely found further outside.

To get a better idea about systematic temperature effects, we computed a characteristic ionization parameter $\xi=L /\left(n r^{2}\right)$, with $L$ being the bolometric luminosity of the ionizing source in the $1-1000$ Rydberg range, $n$ the electron density of the photoionized absorbing gas, and $r$ the distance from the ionizing source. For a grid of ionization parameters, column densities have been computed for each ion using ionization-balance calculations done with Cloudy (Ferland et al. 1998). The SED input into Cloudy consists of IR data from Naik et al. (2009),

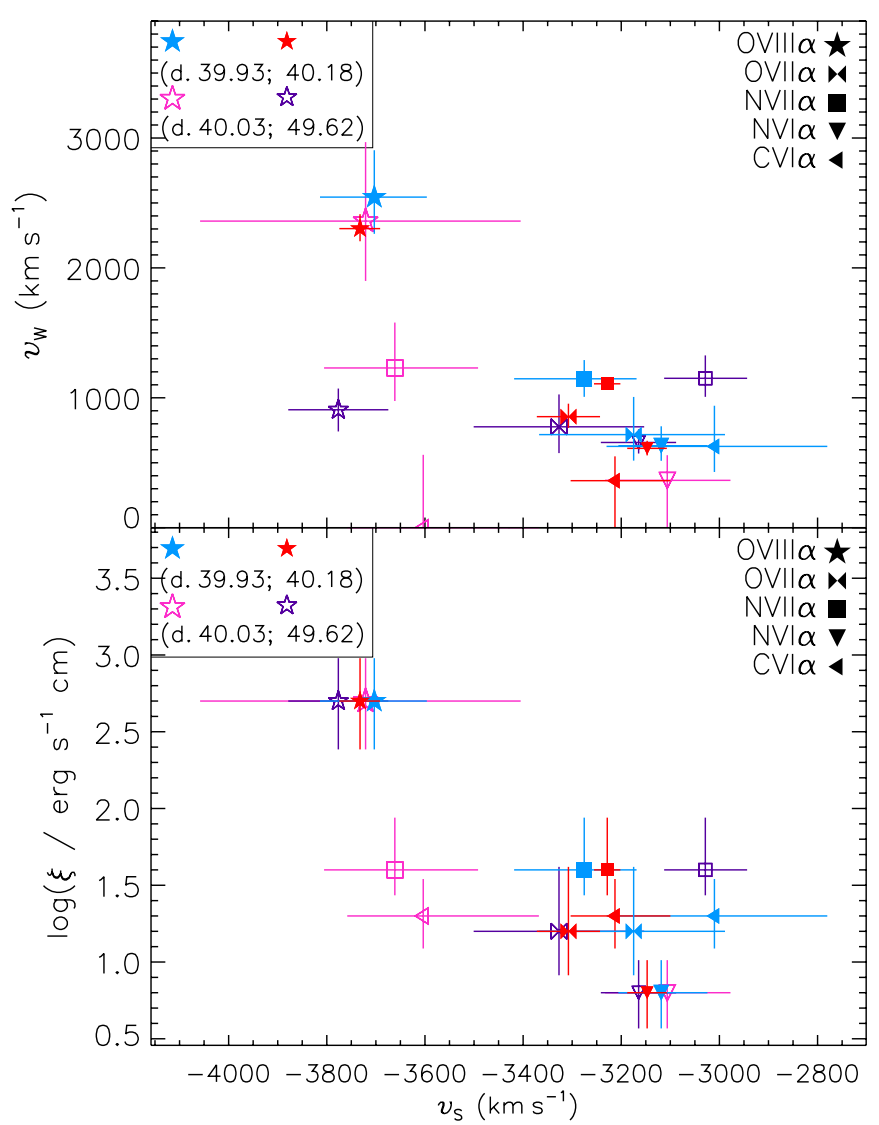

Figure 10. Line shifts plotted vs. line widths (top) and vs. ionization parameter yielding the highest column density (bottom), with the values given in Table 4. The symbols indicate different lines as indicated in the right legend and four symbol colors correspond to the four epochs of extracted spectra (Figure 3 and Table 3).

(A color version of this figure is available in the online journal.)

optical data from the AAVSO database presented by Hachisu \& Kato (2009), UV and soft X-ray fluxes from this paper, and hard X-ray fluxes from Page et al. (2010). Solar abundances by Lodders \& Palme (2009) were assumed. A description of how this was done can be found in Section 6.2 of the SPEX manual ${ }^{15}$ (Kaastra et al. 1996). From the resulting balance curves, the ionization parameter yielding the largest absorption column density for each ion is selected for plotting along the vertical axis in the bottom panel of Figure 10. As a confidence range, the range of ionization parameter is chosen that includes all column densities greater than $10 \%$ below the value at the peak. A trend seems suggestive that lines with higher ionization parameter are more blueshifted, but this conclusion depends very much on the measurements of the $\mathrm{O}$ VIII line. With the given set of lines, it is thus difficult to establish a trend with ionization temperature. A more systematic approach is presented in Pinto et al. (2011).

\subsection{Comparison with Atmosphere Models}

The closest physical approach to derive global parameters of the nova ejecta are non-LTE atmosphere models. Of particular interest for understanding nova evolution are the effective temperature and the mass of the underlying WD. Here we use the publicly available tabulated non-LTE atmosphere models described by Rauch et al. (2010b). The Tübingen Model

\footnotetext{
15 http://www.sron.nl/files/HEA/SPEX/manuals/manual.pdf
} 


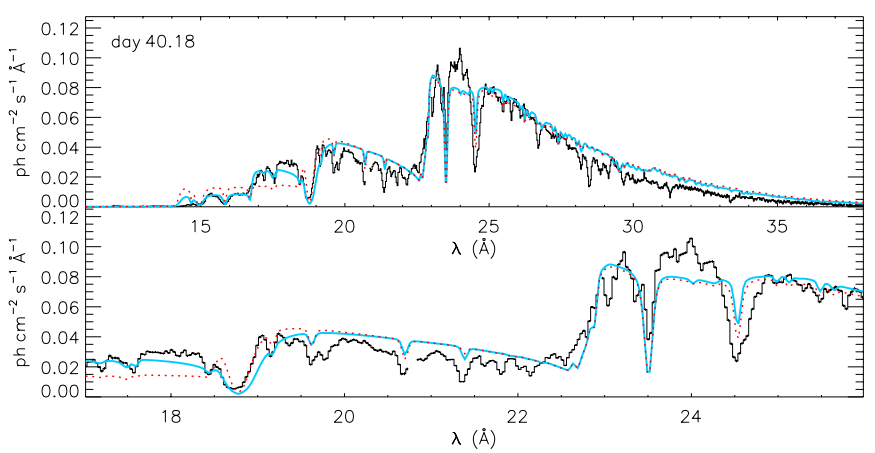

Figure 11. Comparison between model atmosphere and RGS spectrum of V2491 Cyg commencing on day 40.18. Models are taken from Rauch et al. (2010b). Their Table 1 lists different abundance categories, and the blue solid and red dotted lines are models \#007 and \#003, respectively. Both models are blueshifted by $z=-0.01$, corresponding to $v_{\text {shift }}=-3000 \mathrm{~km} \mathrm{~s}^{-1}$. All models assume $\log g=9$.

(A color version of this figure is available in the online journal.)

Atmosphere Package (TMAP) has been developed to study atmospheres of hot WDs, where assumptions such as hydrostatic equilibrium and a plane-parallel geometry are valid approximations. In Section 5.2.1, we discuss in more depth the limitation of these assumptions when applied to novae.

We used all available data tables and obtained best fits with xspec (Arnaud 1996) for all four spectra extracted from the time intervals listed in Table 3. We carried out simultaneous fits to the separate RGS1 and RGS2 spectra and used the fluxed spectra only for plotting. We corrected for photoelectric absorption using the TBnew module developed by Wilms et al. (2000, 2006; J. Wilms et al. 2011, in preparation) with a variable column density of neutral hydrogen, $N_{\mathrm{H}}$, and abundance of neutral oxygen.

The public database currently contains only models assuming $\log g=9$, covering a range of chemical abundances. In Figure 11, the best-fit models \#007 and \#003 are shown with blue solid and red dotted lines, respectively, in comparison to the spectrum from day 40.18. The top panel shows the entire spectrum, and the overall reproduction of the data appears reasonable. The oxygen-rich model \#007 performs better at shorter wavelengths, supporting our earlier conclusion of a high $\mathrm{O}$ abundance. In the bottom panel, a zoom-in is shown, illustrating that some of the absorption lines are represented well. Values of reduced $\chi^{2}$ (number of degrees of freedom in brackets) for model \#007 are 3.7 (5511), 2.2 (5613), 21.8 (5616), and 4.5 (5504), for days 39.93, 40.03, 40.18, and 49.62, respectively. Note that the number of degrees of freedom is overestimated (and thus reduced $\chi^{2}$ is underestimated) because a large number of parameters in the models are not counted as free parameters because they are not varied. Hard-coded values such as NLTE parameters have already been chosen in an early stage of model development and are not further considered during routine fitting, but they would likely change the results if modified. Therefore, they are not necessarily "uninteresting" parameters in the sense of Avni (1976) and may be of importance in deeper studies involving new computations for the special situation of an individual system such as V2491 Cyg. In particular, different values of $\log g=9$ that are currently not publicly available have to be considered, which will significantly increase the parameter space, likely yielding different results.

The model parameters are listed in the right part of Table 3, where the ranges include the best-fit results from using all available models. The ranges do not reflect the full accuracy of physical quantities but only the ranges of parameters if different abundance classes are assumed. It is impossible to predict how the parameters would change if effects of expansion or extended geometry were included. Radii were computed from the model normalization, $R=10^{-11} \times \sqrt{\text { norm }} \times d$, with $d=10.5 \mathrm{kpc}$ being the distance, bolometric luminosities from the Stefan-Boltzmann law, and masses were derived from the radii and $\log g=9, M=R^{2} g / G$ with the gravitational constant $G=6.674281 \times 10^{-8}$ in cgs units.

The parameter of highest interest is the effective temperature, for which we find the same trend with time as already found from the blackbody fits. With the result from the line profiles that the ejecta are extended, allowing us to view through different velocity layers, it is plausible that we are also viewing different temperature layers in the same spectra. Therefore, the definition of a single characteristic temperature does not have the same physical meaning as in a WD evolutionary model and cannot be used in the same way. We also caution that the underlying parameter $\log g=9$ is unrealistic for nova ejecta. The derived masses are completely unrealistic with some values much larger than the Chandrasekhar mass limit. We note that standard mass-radius relations do not apply for active novae since the radiative energy is not drawn from gravitational contraction but from nuclear burning. Also, the bolometric luminosity computed from the Stefan-Boltzmann law, assuming the same radius, is of order $10^{38} \mathrm{erg} \mathrm{s}^{-1}$ and thus also unrealistically high for the late stage of evolution.

It has to be kept in mind that the underlying model assumption is a plane-parallel geometry, and strictly speaking, the calculation of a radius is in violation to this model assumption. Therefore, all calculations of WD masses and luminosities that have been obtained from hydrostatic plane-parallel models have to be treated with greatest caution.

With all these concerns in mind, these models represent the data surprisingly well as can be seen in Figure 11 making it tempting to trust this approach. New data tables with lower $\log g$ may yield more realistic masses, however, spectral fits of models with more realistic parameter combinations have not so far yielded better fits than can be seen in Figure 11 (see, e.g., Nelson et al. 2008; Rauch et al. 2010a).

We argue that a much more detailed discussion of refinement of atmosphere models is required, which is beyond the scope of this paper. While the data tables can be extended to include a larger range of $\log g$, it will in our view be unavoidable that individual models are calculated for each nova, accounting for the individual conditions. Furthermore, more studies are needed to investigate the effects on the derived effective temperature when accounting for the extended geometry and the expansion.

\section{DISCUSSION}

\subsection{Light Curves}

Based on the second half of the first observation, a periodic signal of $2232.1 \mathrm{~s}$ (37.2 minutes, frequency $0.448 \mathrm{mHz}$ ) may be present. We cannot entirely exclude red noise, but it is noteworthy that this signal is of the same order as the $\sim 2500 \mathrm{~s}$ period detected by Drake et al. (2003) in V1494 Aql or the 1300 s period seen by Ness et al. (2003) in V4743 Sgr. It could be the spin period of the WD or originate from pulsations (Drake et al. 2003; Kang et al. 2006). A detailed study of the X-ray light curves of V4743 Sgr by Dobrotka \& Ness (2010) revealed the presence of two nearby signals between days 180 and 196 (see also Leibowitz et al. 2006). While roughly the same period 
was found to be persistent over the course of three years, small frequency changes were found by Dobrotka \& Ness (2010).

During the SSS phase, the frequency changes scaled with changes in the blackbody temperature as predicted by stellar pulsation theory (for details see Dobrotka \& Ness 2010). After day 526, a stable frequency was found in post-outburst $\mathrm{X}$-ray light curves. However, in optical and UV light curves of V4743 Sgr a slightly different frequency was found. They concluded that while pulsations may have been present during the SSS phase, the post-outburst X-ray period could be the WD spin period, modulated by pulsations during the SSS phase. Combined with the orbital period, Kang et al. (2006) and Dobrotka \& Ness (2010) conclude that V4743 Sgr is an intermediate polar. For V2491 Cyg, only two light curves are available, the first of which is interrupted by a dip that has to be excluded from the period analysis. In intermediate polars, the orbital period is usually significantly longer than the spin period of the WD, i.e., there is no spin/orbit synchronization. Baklanov et al. (2008) found a period of $2.3 \mathrm{hr}$ for V2491 Cyg, which is much longer than the 37.2 minute period, but it was not claimed to be the orbital period. The fact that a period can be seen in the X-ray light curve could imply that magnetic fields are involved, but not enough information is available to make a strong enough case for an intermediate polar on these grounds. Particularly puzzling is that the period is not present in the second observation, while the X-ray light curve of an intermediate polar should be modulated with the spin period of the WD. Spectroscopic evidence supporting the interpretation of an intermediate polar is discussed by Takei et al. (2011).

\subsection{Spectra}

\subsubsection{Applicability of Theoretical Stellar Atmospheres}

The ultimate goal of spectral analyses is to determine mass loss, composition, and kinematics. The requirements for finding these parameters are well-exposed X-ray grating spectra that allow individual lines to be resolved and sophisticated spectral models that include the requisite physics and reproduce the observed spectra.

X-ray spectra of CCD resolution $(\sim 100 \mathrm{eV})$ are not sufficient, as spectral models that are different in their details will be indistinguishable after folding them through the instrumental response of a CCD spectrometer. Too many different models can reproduce the same data, yielding non-unique results. Fine tuning of some parameters may improve the value of $\chi^{2}$, but with the limited amount of information in the observed spectrum, the adjustments in the model are arbitrary.

With the XMM-Newton and Chandra gratings, appropriate spectra can be taken and are presented in this paper. The first requirement, well-exposed high-resolution spectra, has been met, however, spectral models need more development. The kinematics in the ejecta are relatively easy to determine from the blueshifts in the absorption lines, but at the same time they pose the biggest challenge to self-consistent non-LTE atmosphere models.

Another problem is that we depend on a very limited number of publicly available models. An attempt has been made by Rauch et al. (2010b) to make available an archive of TMAP models, covering a multi-dimensional space of parameters. While the approach yields the most sophisticated models using a large database of transitions and treating radiation transport in full non-LTE, they are plane-parallel plus static and as such inappropriate for nova ejecta while they are still expanding.
We have shown in Section 4.3, where Table 4 is discussed and Figure 9 is shown as an example, that all photospheric absorption lines are blueshifted as well as significantly broader than the instrumental line broadening function. This can only be interpreted as expanding ejecta that are sufficiently extended to observe a range of layers of different velocity and consequently also temperature. Ness (2010) has shown that this phenomenon can also be observed for other novae. The TMAP models account for non-LTE effects, however, the underlying assumptions of hydrostatic equilibrium and plane-parallel geometry are far from reality for expanding nova ejecta, and our results show that such simplifications are not justified. For more detailed discussion of the importance of the missing physics, we refer to Hauschildt et al. (1992, 1994a, 1994b, 1995) and Hauschildt \& Baron (1999). Although, these arguments apply to an earlier phase of the nova evolution, the high-resolution spectra indicate that the arguments are the same. No models are publicly available that account for all the necessary physics, and we refer to van Rossum \& Ness (2010) who found that atmosphere models that account for the expansion yield vastly different results from static models.

Clearly, atmosphere models are required that can give a full account of the physics in the ejecta without neglecting the extended nature and/or expansion, including the effects of spherical and non-spherical geometry. Without such efforts, no accurate determination of a photospheric temperature is possible, even if the spectra can be well fitted.

Meanwhile, blackbody fits are at most parameterizations yielding no more than a spectral hardness parameter. Some limited, qualitative studies of spectral changes can be carried out, but no quantitative interpretation is possible. The advantage of blackbody fits is the small parameter space, yielding unique parameters, and blackbody temperature values respond sensitively to changes in spectral hardness. While different atmospheric processes may balance out to result in similar temperatures with the brightness decrease on day 40.03, it appears more likely that only the brightness has changed while the atmospheric structure remained the same. This would argue in favor of external reasons for the brightness variations. Meanwhile, the increase in blackbody temperature toward the second observation could be explained by either a higher photospheric temperature or changes in the atmosphere structure. If interpreted as an increase in photospheric temperature, this result would be consistent with a decreasing photospheric radius, exposing successively hotter material.

\subsubsection{Comparison to Other Novae}

V2491 Cyg is the third nova for which a bright SSS X-ray spectrum with atmospheric continuum and deep absorption lines was obtained in high spectral resolution, after V4743 Sgr and RS Oph, and a comparison is instructive.

V4743 Sgr. In Figure 12, a comparison between a calibrated Chandra/LETGS observation of the slower $\left(t_{2}=9\right.$ days; Morgan et al. 2003) nova V4743 Sgr as observed on day 180 after discovery (light blue) and the day 40.18 spectrum of V2491 Cyg (red, see Table 3) is shown. The spectrum of V4743 Sgr is significantly softer than V2491 Cyg, indicating a lower photospheric temperature. While the lower $E(B-V)=0.25$ for V4743 Sgr (as opposed to $E(B-V)=0.43$ for V2491 Cyg by Rudy et al. 2008) could explain a softer X-ray spectrum, the Wien tail being at longer wavelengths makes a strong case for a lower effective temperature. In the bottom panels, the two wavelength ranges $22-27 \AA$ and $27-31 \AA$ are shown in detail. 


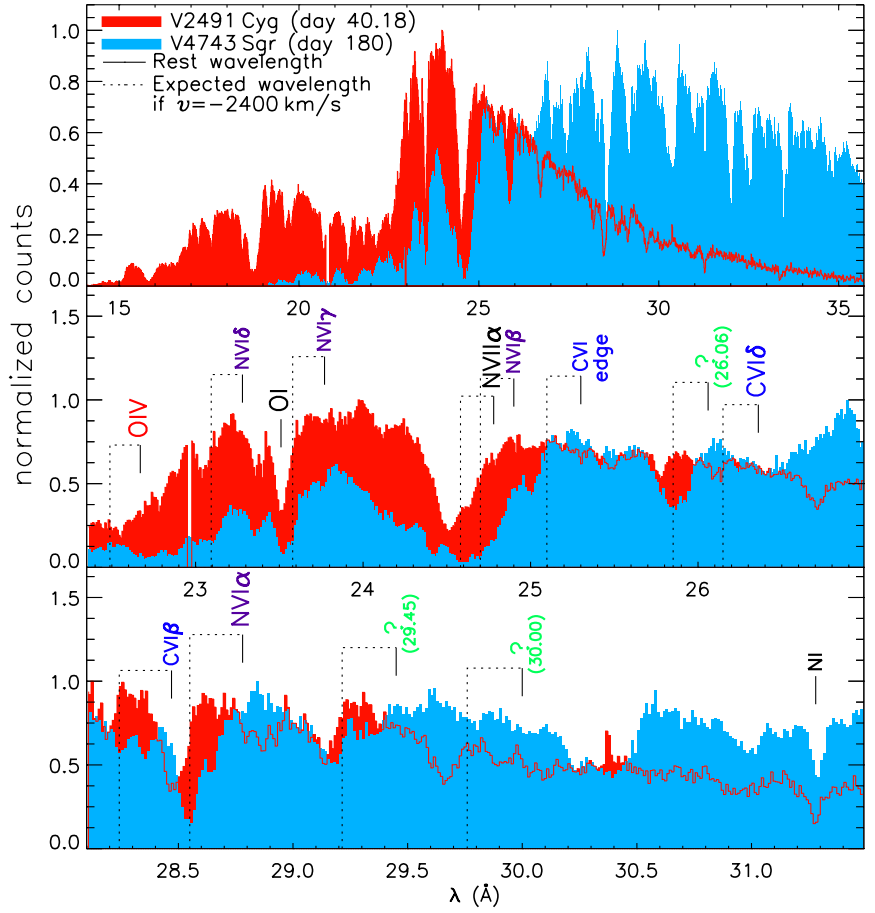

Figure 12. Comparison of a calibrated Chandra/LETGS spectrum of V4743 Sgr (day 180 after discovery; light blue) to the phase c spectrum of V2491 Cyg (starting day 40.18, red as in Figure 3). The same lines as in Figure 7 are marked, and the dotted lines indicate the expected blueshifts for an expansion velocity of $-2400 \mathrm{~km} \mathrm{~s}^{-1}$ (Ness et al. 2003).

(A color version of this figure is available in the online journal.)

The same line labels as those used in Figure 7 are included, but here, we assume a blueshift corresponding to $-2400 \mathrm{~km} \mathrm{~s}^{-1}$, which is consistent with several photospheric lines in V4743 Sgr such as N VII at $25 \AA$ (Ness et al. 2003). The line profiles in V4743 Sgr are much more complex than in V2491 Cyg, some of them consisting of more than one velocity component. A similarly complex situation is encountered in KT Eri (Ness et al. 2010). For example, the line profile around the N vII line is extremely complex in V4743 Sgr, while for V2491 Cyg, only two overlapping lines from N VII and N VI are seen (see Figure 9). The interstellar N I and O I lines at $32.28 \AA$ and $23.5 \AA$, respectively, are present in both novae at the same wavelengths. The O I line blends with the blueshifted N vI $\gamma$ line in V4743 Sgr.

$R S$ Oph. Similar to V2491 Cyg is a grating spectrum taken 39.7 days after the discovery of the 2006 outburst of the recurrent, symbiotic nova RS Oph (Ness et al. 2007) which evolved on a similar timescale $\left(t_{2}=7.9\right.$ days; Hounsell et al. 2010). This is shown in Figure 13 where the calibrated Chandra/ LETGS spectrum of RS Oph (in flux units) was scaled by a factor of 0.4. The observed blueshifts seen in the photospheric lines in RS Oph was $-1200 \mathrm{~km} \mathrm{~s}^{-1}$ (Ness et al. 2007), and the dotted lines mark these projected velocities.

The scaling factor of 0.4 is much larger than is required for the different distances of $1.6 \mathrm{kpc}$ for RS Oph (Bode 1987) and $10.5 \mathrm{kpc}$ for V2491 Cyg. Thus, at the respective times of observation, V2491 Cyg was intrinsically a factor of 17 brighter than RS Oph. However, both novae were extremely variable around the respective times of observations.

The spectrum of V2491 Cyg used for the comparison has been extracted from a phase of bright emission (day 40.18) while the Chandra observation of RS Oph was taken during a time of comparatively low flux (Ness et al. 2007). In addition,

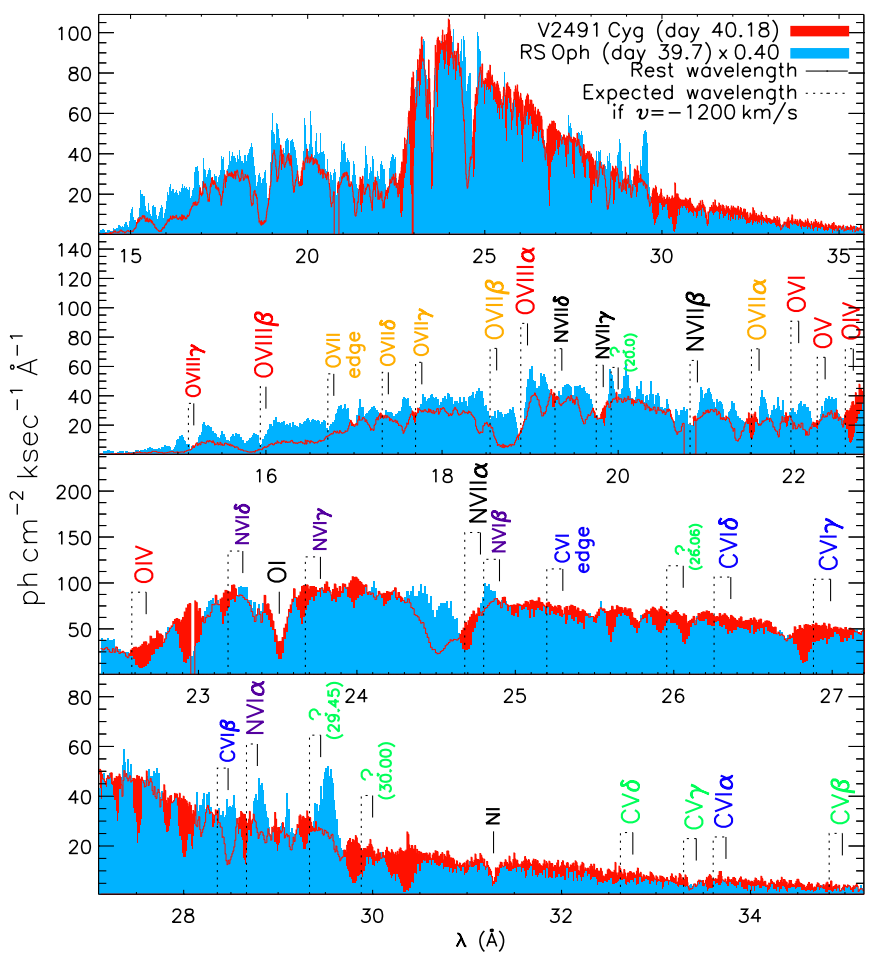

Figure 13. Same as in Figure 12 for RS Oph (light blue). Here line shifts of $-1200 \mathrm{~km} \mathrm{~s}^{-1}$ are marked (Ness et al. 2007).

(A color version of this figure is available in the online journal.)

there are uncertainties in the distance of V2491 Cyg, because the temporary secondary peak seen in V2491 Cyg (see the bottom panel of Figure 1) disallows the use of the MMRD method. Under the assumption of the same luminosity at the respective times of observation, the scaling factor between RS Oph and V2491 Cyg yields a distance of $2.5 \mathrm{kpc}$ for V2491 Cyg. The soft tails of the X-ray spectra of V2491 Cyg and RS Oph are very similar, indicating the same amount of photoelectric absorption, which is consistent with the measurements of $E(B-V)=0.43$ for V2491 Cyg (Rudy et al. 2008; corresponding to $N_{\mathrm{H}}=$ $2.6 \times 10^{21} \mathrm{~cm}^{-2}$ ) and $N_{\mathrm{H}}=2.4 \times 10^{21} \mathrm{~cm}^{-2}$ for RS Oph (Hjellming et al. 1986).

Noteworthy is a remarkable similarity in the continua between the X-ray grating spectra of V2491 Cyg and RS Oph (Figure 13) suggesting a surprisingly similar photospheric temperature at the times of the respective observations. With CCD resolution, V2491 Cyg and RS Oph would be considered twins. However, at grating resolution, small but important differences can be seen, yielding information about the structure of the atmosphere. Most obvious are much smaller blueshifts and line widths of the photospheric lines in RS Oph, indicating slower expansion. Also, the O I absorption edge at $23.5 \AA$ is slightly steeper in V2491 Cyg. While the N VI and N viI lines around 28.7 and $25 \AA$ reach about the same depth in both cases, the O VIII line around $19 \AA$ is much deeper in V2491 Cyg. Since the same depth is reached for the lines of two different ionization stages, $\mathrm{N}$ VI and $\mathrm{N}$ VII, the deeper $\mathrm{O}$ VIII line cannot be a temperature effect, and a higher oxygen abundance in V2491 Cyg appears likely. This could also explain the deeper O I absorption edge. On the other hand, the O VII line at $21.6 \AA$ is only slightly deeper in V2491 Cyg, but that could be explained by photoionization from $\mathrm{O}$ VII to $\mathrm{O}$ VIII, which is supported by the presence of the O VII absorption edge at $16.7 \AA$ in V2491 Cyg (see Figure 8) that seems less pronounced in RS Oph. 
Table 5

List of Unidentified Lines

\begin{tabular}{lcccc}
\hline \hline$\lambda_{\text {proj }}{ }^{\mathrm{a}}(\AA)$ & \multicolumn{3}{c}{$\lambda_{\text {obs }}(\AA)$} & Comment \\
& V2491 Cyg & V4743 Sgr & RS Oph & \\
\hline 20.00 & 19.80 & $\ldots$ & $\ldots$ & Low- $T$ line \\
25.70 & $\ldots$ & $\ldots$ & 25.6 & \\
25.80 & $\ldots$ & $\ldots$ & 25.7 & \\
26.06 & 25.78 & 25.85 & 25.90 & Low- $T$ line \\
26.21 & $\ldots$ & $\ldots$ & 26.1 & \\
26.93 & $\ldots$ & $\ldots$ & 26.83 & \\
27.41 & $\ldots$ & $\ldots$ & 27.3 & \\
27.61 & $\ldots$ & $\ldots$ & 27.5 & \\
27.71 & $\ldots$ & $\ldots$ & 27.6 & \\
28.11 & $\ldots$ & 29.18 & 28.0 & \\
29.45 & 29.13 & $\ldots$ & 29.30 & High- $T$ line \\
30.00 & 29.66 & $\ldots$ & 29.70 & High- $T$ line \\
30.42 & $\ldots$ & & 30.3 & \\
\hline
\end{tabular}

Note. ${ }^{a}$ Projected, =assumed rest wavelength, assuming blueshifts of $3300 \mathrm{~km} \mathrm{~s}^{-1}$ for V2491 Cyg, $2400 \mathrm{~km} \mathrm{~s}^{-1}$ for V4743 Sgr, and $1200 \mathrm{~km} \mathrm{~s}^{-1}$ for RS Oph.

Unidentified lines. While the optical evolution was different, yielding a rebrightening in V2491 Cyg that was not seen in RS Oph, V2491 Cyg was suggested to be an RN (Pagnotta et al. 2009; Page et al. 2010; Ragan et al. 2011), and the spectral similarity to one of the most famous RNe, RS Oph, may be an additional indicator.

While the measurement of line shifts relies on well-identified lines, a number of absorption lines cannot be identified. The comparison with other novae gives us an idea of the formation conditions of unidentified lines, aiding future identification efforts. At $25.85 \AA$, a broad line is seen in V4743 Sgr that coincides with a line at $26.06 \AA$ that is marked as unidentified in Figure 7. With the lower photospheric temperature of V4743 Sgr, this line could be formed at relatively lower temperature since it is much more pronounced in V4743 Sgr. Another unidentified line in Figure 7, with a projected rest wavelength of $29.45 \AA$, is also present in V4743 Sgr with roughly the same width and strength. The only difference is the line shift, which is similar to the N VI line at $28.8 \AA$. This line might be formed at a slightly higher temperature than N VI which is deeper in V4743 Sgr. The line at $30 \AA$ projected rest wavelength is not present in V4743 Sgr and could be formed at temperatures that are higher than in V4743 Sgr. The unidentified lines are listed in Table 5 with projected rest wavelengths in the first column and observed wavelengths for V2491 Cyg, V4743 Sgr, and RS Oph (see below), respectively.

The unidentified lines with projected rest wavelengths 26.06 , 29.45, and 30.0 $\AA$ are also present in RS Oph. The $26.06 \AA$ line is weaker in RS Oph than in V4743 Sgr, but stronger than in V2491 Cyg, while the $29.45 \AA$ line has about the same strength. This supports the conclusion that the $26.06 \AA$ line is a low-temperature line while the $29.45 \AA$ line is formed at higher temperatures. In contrast to V4743 Sgr, the $30.0 \AA$ line is clearly present in RS Oph and is deeper than in V2491 Cyg. Additional unidentified lines are seen in RS Oph, e.g., at 25.6, $25.7,26.1,27.3,27.5,27.6,28.0$, and $30.3 \AA$, but these lines are not seen in V2491 Cyg and V4743 Sgr. Two of the unidentified lines are seen in all three novae at wavelengths yielding the same blueshifts as other photospheric lines. Meanwhile, a large number of lines are only present in the spectrum of RS Oph, arising in the same wavelength range as a pre-SSS emission

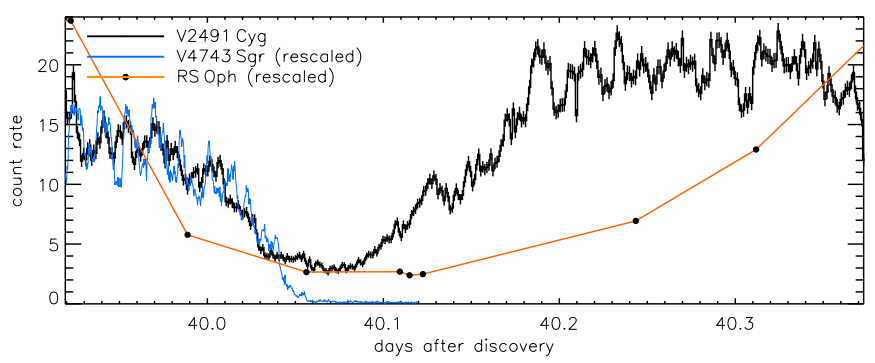

Figure 14. Comparison of the X-ray light curve of V2491 Cyg after day 39.6 with rescaled Chandra and Swift light curves of V4743 Sgr with the decay on day 180.6 and of RS Oph with one of the minima reported by Osborne et al. (2011), respectively.

(A color version of this figure is available in the online journal.)

line component around day 26 (Ness et al. 2009b; Nelson et al. 2008).

The emission lines in the RS Oph spectrum at 28.8, 29.1, and $29.5 \AA$ originate from the N vi He-like triplet. These and other components of the red wings of absorption lines such as $\mathrm{O}$ VIII $(18.97 \AA)$ and N VII $(24.8 \AA)$ are related to the P-Cygni-like profiles discussed by Ness et al. (2007). They could partially originate in shocked plasma and might not be related to the atmospheric emission.

Other novae. Other nova SSS spectra such as those of V1494 Aql (Rohrbach et al. 2009) are more complicated and/or less well exposed. All high-quality SSS spectra show significant blueshifts in their absorption lines, and the conclusion is unavoidable that expansion continues into the SSS phase (Ness 2010). It is noteworthy that the expansion velocities can be quite different, yielding the lowest expansion velocity in RS Oph, even though the spectral shape of the continuum and the depths of most absorption lines are remarkably similar to V2491 Cyg. The lower expansion velocity in RS Oph may be explained by the presence of a dense stellar wind of the companion that has absorbed some of the kinetic energy during the earlier phases of the outburst. This results in significant deceleration occurring by $\sim 40$ days after outburst (e.g., Bode et al. 2006). While some evidence for the companion in V2491 Cyg to be an evolved object is presented by Ribeiro et al. (2011), the mass-loss rate is likely to be much lower than in RS Oph. Meanwhile, in V4743 Sgr, some of the absorption lines contain at least three velocity components while V2491 Cyg and RS Oph contain only one velocity component. Since V4743 Sgr is a slower nova, it may be possible that the ejecta in slower novae are generally more structured than those of faster novae. This would be consistent with modeling of common envelope interaction (Lloyd et al. 1997; Porter et al. 1998), but other slower novae need to be studied for firmer conclusions.

\subsubsection{High-amplitude Variations}

During the earlier of the two XMM-Newton observations, lasting from day 39.93 to 40.37 , V2491 Cyg was highly variable in X-rays, including a $3 \mathrm{hr}$ dip. Several novae have been observed with an episode of high-amplitude variations during their early SSS phase, but an explanation is still pending. The first observed occurrence of a sudden decay in the X-ray output of a nova was encountered in a Chandra observation of V4743 Sgr (see Figure 14 and Ness et al. 2003). The count rate declined almost to zero, leaving behind an emission line spectrum, originating from photoionization plasma with some emission lines that were rapidly declining (J. U. Ness et al. 2011, in preparation). 
At that time, no monitoring was possible, and the nova did not rebrighten during the same observation. Two weeks later, the nova was bright again, but highly variable (Leibowitz et al. 2006). The dip in the first XMM-Newton observation of V2491 Cyg occurred on the same timescale as the decay in V4743 Sgr, as illustrated in Figure 14, and the same phenomenon may have been observed.

Dense Swift monitoring of RS Oph revealed multiple declines (Osborne et al. 2011) which occurred on timescales that are also similar to V2491 Cyg (Figure 14). This phenomenon has not been fully explained yet. One suggestion is temporary photospheric expansion, which would be supported by the disappearance of periodic oscillations during the dip which likely originate from close to the WD. Such deep dips could also mean that the concept of constant bolometric luminosity is wrong as suggested by Starrfield et al. (2009). However, intrinsic changes in bolometric luminosity are not necessarily easier to understand than changes in the structure of the ejecta. A change in luminosity would imply a change in the ionization parameter, $\xi=L /\left(n r^{2}\right)$, of the photoionized gas, with a subsequent evolution of the photoionization balance. Since the density of the photoionized absorbing gas, $n$, and distance to ionizing source, $R$, are unlikely to change significantly, intrinsic changes of the bolometric luminosity would have lead to equally significant changes in the ionization parameter. A detailed study using photoionization models is in progress (Pinto et al. 2011).

\section{SUMMARY AND CONCLUSIONS}

A small sample of extremely well-exposed grating spectra of novae during their SSS phase has been gathered. The fast CN V2491 Cyg was observed twice during its extremely short SSS phase, covering two different phases of evolution. The first observation was taken early while the X-ray brightness was highly variable, containing a deep dip, while the second observation was taken during the decline with a lower count rate and less variability. The dip evolved on a timescale similar to the decay in the slower nova V4743 Sgr (Ness et al. 2003) and the variations during the early SSS phase in RS Oph (Osborne et al. 2011). High-amplitude variations seem to be a common phenomenon that has now been observed in many novae.

The contemporaneous UV light curves show short-term variability, but no UV data were taken during the dip in the first X-ray light curve. A 37.2 minute period was detected in $\mathrm{X}$-rays before and after but not during the dip. It could reflect the WD spin period, but the statistical significance is not high.

The large majority of absorption lines in the high-resolution RGS X-ray spectra yield a single velocity component with blueshifts ranging between -3000 and $-3400 \mathrm{~km} \mathrm{~s}^{-1}$. Additional interstellar lines are found at their rest wavelengths. A few deep absorption lines are currently not identified. The same lines are also present in the SSS spectra of V4743 Sgr and RS Oph at a different blueshift, indicating that they are of photospheric origin.

The narrow range of blueshifts is different from V4743 Sgr where at least three velocity components can be seen. This could be explained by the slower evolution of V4743 Sgr. The SSS spectrum of RS Oph is remarkably similar in the shape of the continuum, yet is very different in the properties of the absorption lines, yielding much smaller blueshifts. The lower velocities in RS Oph may be due to the presence of the pre-outburst red giant wind, while the higher blueshifts in V2491 Cyg could be the explanation for the short duration of the SSS phase.
Archived atmosphere models yield good fits to the SSS spectra, but the model parameters are unphysical. This can be explained by the simplifications in the model, assuming hydrostatic equilibrium and a compact atmosphere without expansion.

The conclusions are the following.

1. Significant blueshifts in all photospheric absorption lines demonstrate that the nova ejecta are expanding.

2. Significant line broadening of most photospheric absorption lines demonstrates that the ejecta are extended, exposing a range of velocity layers and thus also temperature layers.

3. Our conclusions from line profile analysis indicate that publicly available stellar atmosphere models do not contain enough physics for nova ejecta, even though they yield surprisingly good fits to the data.

4. Comparison with other novae of lower and higher photospheric temperatures allows us to spot so far unknown nova absorption lines and associate them to higher or lower temperature environments.

5. Remarkable spectral similarities between V2491 Cyg and RS Oph indicate similar system characteristics. Assuming the same luminosities, the distance to V2491 Cyg would only be $2.5 \mathrm{kpc}$.

6. The different properties of the absorption lines between V2491 Cyg and RS Oph indicate that similar global parameters can still lead to a different atmospheric structure.

7. Slower novae such as V4743 Sgr may contain more complex absorption line systems than faster novae such as RS Oph or V2491 Cyg.

8. The single dip in the X-ray light curve occurred on the same timescale as in V4743 Sgr and RS Oph, and the underlying process may therefore be common to all novae in which an early variation phase is observed in X-rays.

We thank the XMM-Newton SOC team for their support in scheduling and analyzing these TOO observations. Special thanks to Aitor Ibarra who supported us with the new SAS tool xmmextractor. We acknowledge with thanks the variable star observations from the AAVSO International Database contributed by observers worldwide and used in this research. J.-U.N. gratefully acknowledges support provided by NASA through Chandra Postdoctoral Fellowship grant PF5-60039, awarded by the Chandra X-ray Center, which is operated by the Smithsonian Astrophysical Observatory for NASA under contract NAS8-03060. A.D. and C.P. acknowledge financial support from the Faculty of the European Space Astronomy Centre. A.D. was supported in part by the Grand-in-Aid for the global COE programs on "The Next Generation of Physics, spun from Diversity and Emergence" from MEXT and also by the Slovak Grant Agency, grant VEGA-1/0520/10. K.L.P., A.P.B., and J.P.O. acknowledge support from STFC. J.J.D. was supported by NASA contract NAS8-39073 to the Chandra X-ray Center during the course of this research. SRON Netherlands is supported financially by NWO, The Netherlands Organization for Scientific Research. S.S. acknowledges support from NASA+NSF grants to ASU. We thank Rick Rudy and Dave Lynch for the information on the peak at the later NIR spectra. M.H. acknowledges support from MICINN project AYA 200801839/ESP, AGAUR project 2009 SGR 315 and FEDER funds, and GS MICINN projects AYA 2008-04211-C02-01 and AYA 2007-66256.

Facilities: AAVSO 


\section{REFERENCES}

Arnaud, K. A. 1996, in ASP Conf. Ser. 101, Astronomical Data Analysis Software and Systems V, ed. G. H. Jacoby \& J. Barnes (San Francisco, CA: ASP), 17

Avni, Y. 1976, ApJ, 210, 642

Baklanov, A., Pavlenko, E., \& Berezina, E. 2008, ATel, 1514, 1

Bode, M. F. (ed.) 1987, RS Ophiuchi (1985) and the Recurrent Nova Phenomenon (Utrecht: VNU Science), 241

Bode, M. F., \& Evans, A. (ed.) 2008, Classical Novae (Cambridge: Cambridge Univ. Press)

Bode, M. F., et al. 2006, ApJ, 652, 629

Bohlin, R. C., Savage, B. D., \& Drake, J. F. 1978, ApJ, 224, 132

della Valle, M., \& Livio, M. 1995, ApJ, 452, 704

den Herder, J. W., et al. 2001, A\&A, 365, L7

Dickey, J. M., \& Lockman, F. J. 1990, ARA\&A, 28, 215

Dobrotka, A., \& Ness, J. 2010, MNRAS, 405, 2668

Downes, R. A., \& Duerbeck, H. W. 2000, AJ, 120, 2007

Drake, J. J., et al. 2003, ApJ, 584, 448

Ferland, G. J., Korista, K. T., Verner, D. A., Ferguson, J. W., Kingdon, J. B., \& Verner, E. M. 1998, PASP, 110, 761

Gallagher, J. S., \& Starrfield, S. 1978, ARA\&A, 16, 171

Grevesse, N., \& Sauval, A. J. 1998, Space Sci. Rev., 85, 161

Hachisu, I., \& Kato, M. 2009, ApJ, 694, L103

Hauschildt, P. H., \& Baron, E. 1999, J. Comput. Appl. Math., 109, 41

Hauschildt, P. H., Starrfield, S., Austin, S., Wagner, R. M., Shore, S. N., \& Sonneborn, G. 1994a, ApJ, 422, 831

Hauschildt, P. H., Starrfield, S., Shore, S. N., Allard, F., \& Baron, E. 1995, ApJ, 447,829

Hauschildt, P. H., Starrfield, S., Shore, S. N., Gonzalez-Riestra, R., Sonneborn, G., \& Allard, F. 1994b, AJ, 108, 1008

Hauschildt, P. H., Wehrse, R., Starrfield, S., \& Shaviv, G. 1992, ApJ, 393, 307

Helton, L. A., Woodward, C. E., Vanlandingham, K., \& Schwarz, G. J. 2008, CBET, 1379, 1

Hernanz, M., \& Sala, G. 2002, Science, 298, 393

Hjellming, R. M., van Gorkom, J. H., Seaquist, E. R., Taylor, A. R., Padin, S., Davis, R. J., \& Bode, M. F. 1986, ApJ, 305, L71

Horne, J. H., \& Baliunas, S. L. 1986, ApJ, 302, 757

Hounsell, R., et al. 2010, ApJ, 724, 480

Ibarra, A., \& Kuulkers, E. 2008, ATel, 1473, 1

Ibarra, A., et al. 2009, A\&A, 497, L5

Kaastra, J. S., Mewe, R., \& Nieuwenhuijzen, H. 1996, in UV and X-ray Spectroscopy of Astrophysical and Laboratory Plasmas, ed. K. Yamashita \& T. Watanabe (Tokyo: Univ. Ac. Press), 411

Kahabka, P., \& van den Heuvel, E. P. J. 1997, ARA\&A, 35, 69

Kang, T. W., Retter, A., Liu, A., \& Richards, M. 2006, AJ, 132, 608

Krautter, J., Ögelman, H., Starrfield, S., Wichmann, R., \& Pfeffermann, E. 1996, ApJ, 456, 788

Kuulkers, E., et al. 2008, ATel, 1480, 1

Landi, E., Del Zanna, G., Young, P. R., Dere, K. P., Mason, H. E., \& Landini, M. 2006, ApJS, 162, 261

Leibowitz, E., Orio, M., Gonzalez-Riestra, R., Lipkin, Y., Ness, J.-U., Starrfield, S., Still, M., \& Tepedelenlioglu, E. 2006, MNRAS, 371, 424

Lloyd, H. M., O’Brien, T. J., \& Bode, M. F. 1997, MNRAS, 284, 137

Lloyd, H. M., O’Brien, T. J., Bode, M. F., Predehl, P., Schmitt, J. H. M. M., Truemper, J., Watson, M. G., \& Pounds, K. A. 1992, Nature, 356, 222

Lodders, K., \& Palme, H. 2009, Meteorit. Planet. Sci. Suppl., 72, 5154
Morgan, G. E., Ringwald, F. A., \& Prigge, J. W. 2003, MNRAS, 344, 521

Mukai, K., \& Ishida, M. 2001, ApJ, 551, 1024

Munari, U., Siviero, A., Dallaporta, S., Cherini, G., Valisa, P., \& Tomasella, L. 2011, New Astronomy, 16, 209

Naik, S., Banerjee, D. P. K., \& Ashok, N. M. 2009, MNRAS, 394, 1551

Nakano, S., et al. 2008, IAU Circ., 8934, 1

Nelson, T., Orio, M., Cassinelli, J. P., Still, M., Leibowitz, E., \& Mucciarelli, P. 2008, ApJ, 673, 1067

Ness, J.-U. 2010, Astron. Nachr., 331, 179

Ness, J.-U., Drake, J. J., Starrfield, S., Bode, M., Page, K., Beardmore, A., Osborne, J. P., \& Schwarz, G. 2010, ATel, 2418, 1

Ness, J.-U., et al. 2003, ApJ, 594, L127

Ness, J.-U., et al. 2007, ApJ, 665, 1334

Ness, J.-U., et al. 2008a, ATel, 1561, 1

Ness, J.-U., et al. 2008b, ATel, 1573, 1

Ness, J.-U., et al. 2009a, AJ, 137, 4160

Ness, J.-U., et al. 2009b, AJ, 137, 3414

Orio, M., et al. 2001, MNRAS, 326, L13

Osborne, J. P., et al. 2011, ApJ, 727, 124

Page, K. L., Osborne, J. P., Beardmore, A. P., Goad, M. R., Wynn, G. A., Bode, M. F., \& O’Brien, T. J. 2008, in ASP Conf. Ser. 401, RS Ophiuchi (2006) and the Recurrent Nova Phenomenon, ed. A. Evans, M. F. Bode, T. J. O'Brien, \& M. J. Darnley (San Francisco, CA: ASP), 283

Page, K. L., et al. 2010, MNRAS, 401, 121

Pagnotta, A., Schaefer, B. E., Xiao, L., Collazzi, A. C., \& Kroll, P. 2009, AJ, 138,1230

Pinto, C., et al. 2011, A\&A, submitted

Porter, J. M., O’Brien, T. J., \& Bode, M. F. 1998, MNRAS, 296, 943

Ragan, E., et al. 2011, in ASP Conf. Ser., in press, arXiv:1004.0419

Rauch, T., Orio, M., Gonzales-Riestra, R., Nelson, T., Still, M., Werner, K., \& Wilms, J. 2010a, ApJ, 717, 363

Rauch, T., Ringat, E., \& Werner, K. 2010b, in Proc. Physics of Accretion Compact Objects Conference, arXiv:1011.3628

Ribeiro, V. A. R. M., Darnley, M. J., Bode, M. F., Munari, U., Harman, D. J., Steele, I. A., \& Meaburn, J. 2011, MNRAS, 412, 1701

Rohrbach, J. G., Ness, J., \& Starrfield, S. 2009, AJ, 137, 4627

Rudy, R. J., Lynch, D. K., Russell, R. W., Woodward, C. E., \& Covey, K. 2008, IAU Circ., 8938, 2

Scargle, J. D. 1982, ApJ, 263, 835

Starrfield, S., et al. 2009, in Conf. on Chandra's First Decade of Discovery, 2009 September 22-25, Boston, MA, ed. S. Wolk, A. Fruscione, \& D. Swartz, abstract 199

Takei, D., Tsujimoto, M., Kitamoto, S., Ness, J., Drake, J. J., Takahashi, H., \& Mukai, K. 2009, ApJ, 697, L54

Takei, D., et al. 2011, PASJ, in press

Talavera, A. 2009, Ap\&SS , 320, 177

Tomov, T., Mikolajewski, M., Brozek, T., Ragan, E., Swierczynski, E., Wychudzki, P., \& Galan, C. 2008a, ATel, 1485, 1

Tomov, T., Mikolajewski, M., Ragan, E., Swierczynski, E., \& Wychudzki, P. 2008b, ATel, 1475, 1

van Rossum, D., \& Ness, J.-U. 2010, Astron. Nachr., 331, 175

Vaughan, S. 2005, A\&A, 431, 391

Vaughan, S. 2010, MNRAS, 402, 307

Warner, B. 2008, in Classical Novae, ed. M. Bode \& A. Evans (Cambridge: Cambridge Univ. Press), 16

Wesson, R., et al. 2008, ApJ, 688, L21

Wilms, J., Allen, A., \& McCray, R. 2000, ApJ, 542, 914

Wilms, J., Juett, A., Schulz, N., \& Nowak, M. 2006, BAAS, 38, 376 